\title{
Signaling defects associated with insulin resistance in nondiabetic and diabetic individuals and modification by sex
}

\author{
Nida Haider, ${ }^{1}$ Jasmin Lebastchi, ${ }^{1,2}$ Ashok Kumar Jayavelu, ${ }^{3}$ Thiago M. Batista, ${ }^{1}$ Hui Pan, ${ }^{4}$ Jonathan M. Dreyfuss, ${ }^{4}$ \\ Ivan Carcamo-Orive, ${ }^{5}$ Joshua W. Knowles, ${ }^{5}$ Matthias Mann, ${ }^{3}$ and C. Ronald Kahn ${ }^{1}$
}

${ }^{1}$ Section of Integrative Physiology and Metabolism, Joslin Diabetes Center, Harvard Medical School, Boston, Massachusetts, USA. ²Division of Endocrinology, Brown, Alpert Medical School, Providence, Rhode Island, USA. ${ }^{3}$ Department of Proteomics and Signal Transduction, Max Planck Institute of Biochemistry, Martinsried, Germany. ${ }^{4}$ Bioinformatics and Biostatistics Core, Joslin Diabetes Center, Harvard Medical School, Boston, Massachusetts, USA. 5Division of Cardiovascular Medicine, Cardiovascular Institute and Diabetes Research Center, Stanford University School of Medicine, Stanford, California, USA.

Insulin resistance is present in one-quarter of the general population, predisposing these people to a wide range of diseases. Our aim was to identify cell-intrinsic determinants of insulin resistance in this population using induced pluripotent stem cell-derived (iPSC-derived) myoblasts (iMyos). We found that these cells exhibited a large network of altered protein phosphorylation in vitro. Integrating these data with data from type 2 diabetic iMyos revealed critical sites of conserved altered phosphorylation in IRS-1, AKT, mTOR, and TBC1D1 in addition to changes in protein phosphorylation involved in Rho/ Rac signaling, chromatin organization, and RNA processing. There were also striking differences in the phosphoproteome in cells from men versus women. These sex-specific and insulin-resistance defects were linked to functional differences in downstream actions. Thus, there are cell-autonomous signaling alterations associated with insulin resistance within the general population and important differences between men and women, many of which also occur in diabetes, that contribute to differences in physiology and disease.

\section{Introduction}

Insulin resistance is a major risk factor in the development of metabolic syndrome, type 2 diabetes (T2D), and cardiovascular disease (1-4). Indeed, the cardiometabolic syndrome currently affects $20 \%$ to $30 \%$ of Westernized populations, and its prevalence continues to increase worldwide, with differing presentations in an age- and sex-specific manner (5). Although the impact of insulin resistance on glucose homeostasis and metabolic syndrome is well studied, $20 \%$ to $30 \%$ of nondiabetic people within the general population also have a substantial level of insulin resistance, and the molecular determinants underlying the insulin resistance in this population remain elusive $(6,7)$. In individuals with a family history of T2D, insulin resistance precedes and predicts a high risk of developing the disease (4), whereas in individuals without a family history of diabetes, insulin resistance appears to be linked to increased risk for hyperlipidemia and accelerated atherosclerosis (8), but not necessarily diabetes (9).

At the cellular level, insulin signaling is initiated by ligand binding leading to conformational change and transautophos-

\section{Delated Commentary: https://doi.org/10.1172/JCl154699}

Authorship note: $\mathrm{NH}, \mathrm{JL}$, and $\mathrm{AKJ}$ are co-first authors.

Conflict of interest: The authors have declared that no conflict of interest exists. Copyright: @ 2021, American Society for Clinical Investigation.

Submitted: May 27, 2021; Accepted: August 26, 2021; Published: November 1, 2021.

Reference information: J Clin Invest. 2021;131(21):e151818.

https://doi.org/10.1172/JCl151818. phorylation of the insulin receptor (IR), which leads to activation of the receptor and phosphorylation of IR substrates, such as the IRS proteins and Shc. As a result, 2 major downstream signaling cascades are initiated: the Ras/MAP kinase pathway and the PI3K/Akt pathway $(10,11)$. Insulin signaling also activates serine/ threonine protein kinase mTOR C1 (mTORC1) to regulate protein translation and cell growth (12), stimulates glucose transport (13), inactivates glycogen synthase kinase 3 (GSK3), regulating glycogen synthesis (14), activates atypical PKCs mediating lipid metabolism (15), and leads to phosphorylation of FoxO1 and FoxK1/ FoxK2 $(16,17)$, which serve as transcriptional regulators of insulin action. The insulin-signaling events play an important role in the regulation of cellular metabolism and growth in the classical insulin-responsive tissues, such as the muscle, liver, and adipose tissue $(10,18)$. Given that skeletal muscle is the largest organ in the body and the primary site of glucose disposal, skeletal muscle insulin resistance largely contributes to dysregulation of whole-body glucose homeostasis (reviewed in ref. 19). The goal of the current study was to investigate the cell-autonomous determinants of insulin resistance and phosphorylation-mediated signaling within the nondiabetic population using myoblasts derived from induced pluripotent stem cells (iPSCs) in vitro.

In this study, we show that iPSC-derived myoblasts (iMyos) from nondiabetic individuals in the highest quintile of insulin resistance show defective insulin-stimulated glucose uptake as compared with iMyos from the most insulin-sensitive quintile, thus replicating the in vivo insulin resistance defects in vitro. Quantitative global phosphoproteomic analysis of these cells reveals a large 
network of proteins whose phosphorylation is altered in association with insulin resistance in both the basal and insulin-stimulated states. A fraction of these alterations is also found in iMyos from T2D patients, thus defining an important core set of defects in cellular signaling in insulin resistance in both nondiabetic and diabetic individuals. Surprisingly, we also observed phosphorylation differences in the iMyos taken from men versus women, which were reflected in sex-dependent differences in downstream cellular effects. These findings provide insights into the cellautonomous mechanisms associated with insulin resistance and demonstrate that these differences are further modified by sex and can be observed in vitro in the absence of hormones and circulating extrinsic drivers of insulin resistance.

\section{Results}

Insulin-resistant nondiabetic iPSC cohort metabolic characterization. Human iPSCs and myoblasts derived from these iPSCs taken from patients with IR mutations and T2D have revealed the power of this system to identify genetic and cell-autonomous components of insulin resistance ex vivo, i.e., in the absence of the multiple circulating factors that induce insulin resistance (20-23). In the present study, we have utilized this approach to study the cellautonomous determinants of insulin resistance within the general population. iPSCs from 10 individuals in the top quintile of insulin sensitivity (I-Sen) and 10 nondiabetic individuals in the bottom quintile, i.e., most insulin resistant (I-Res), previously identified by population screening using the steady-state plasma glucose approach (SSPG) (24), were used (Figure 1A). Both the I-Sen and I-Res cohorts were equally divided between men and women (Supplemental Figure 1A; supplemental material available online with this article; https://doi.org/10.1172/JCI151818DS1) and had an average age of approximately 60 (range 41-79 years; Figure 1B). I-Res subjects had slightly higher BMIs and fasting plasma glucose levels than I-Sen subjects (Figure 1, B and C). The most striking difference, however, was the differential insulin-resistance status as assessed by SSPG, with I-Res subjects having a mean steadystate plasma glucose of $216 \pm 10.3 \mathrm{mg} / \mathrm{dL}$ as compared with $69 \pm$ $6.5 \mathrm{mg} / \mathrm{dL}$ in the I-Sen subjects (Figure 1C).

The iPSCs were derived from blood cells of these individuals using nonintegrative Sendai virus (24) and converted to iMyos using a 2-stage cocktail approach (25). Myogenic differentiation capacity was not affected by insulin-resistance status or sex of the donor, as shown by comparable levels of MyoD1 nuclear staining (Supplemental Figure 1B). In addition, mRNA analysis of the iMyos showed changes in gene expression with differentiation across all cell lines, with marked downregulation of the pluripotency markers NANOG, SOX2, DNMT3B, and POU5F1 and significant upregulation of the myoblast markers $P A X 7, P A X 3, M Y O G$, and MYOD1 (Supplemental Figure 1C). Assessment of glucose uptake using $2\left[{ }^{14} \mathrm{C}(\mathrm{U})\right]$-deoxy-D-glucose (2-DOG) showed an approximately $30 \%$ increase in insulin-stimulated glucose uptake in the I-Sen iMyos, typical of cultured myoblasts (26), and this was significantly impaired in I-Res myoblasts (Figure 1D and Supplemental Figure 1D). Western blot analysis of iMyos following insulin stimulation revealed comparable increases in phosphorylation of IR $\beta^{\mathrm{Y} 1150 / 1151}$ and $\mathrm{AKT}^{\mathrm{S} 473}$ in the I-Sen and I-Res myoblasts, but a significant $10 \%$ to $38 \%$ reduction in insulin-stimulated phosphorylation of
GSK3 $\alpha /$ s21/9 in I-Res men and women (Figure 1, E and F). Taken together, these data demonstrate that differentiated iMyos are insulin responsive and that cells from insulin-resistant individuals mirror the presence of mild to moderate insulin resistance in vitro.

Insulin-regulated phosphoproteome profile. To identify the full spectrum of cellular signaling changes associated with the differences in insulin sensitivity, we performed a global phosphoproteomics analysis of the I-Sen and I-Res iMyos with or without insulin stimulation using liquid chromatography-tandem mass spectrometry (LC-MS/MS) (Supplemental Figure 2A). Between 14,000 and 16,000 phosphosites with a localization probability of more than $75 \%$ (average $=97 \%$ ) could be quantitated in each of the cell lines (Supplemental Figure 2B). Principal component analysis (PCA) of the entire data set demonstrated a clear separation based on 2 factors, with component 1 (the largest driver of variance in the data) being the sex of the cell donor and component 2 (the second largest driver) being the donor's insulin-sensitivity status (Figure 2A). Interestingly, in men, insulin-resistance status shifted the relative coordinates upwards and to the left (filled squares vs. open squares), whereas in women, insulin resistance shifted the coordinates toward the right (filled circles vs. open circles) in the PCA plot, suggesting an interaction between insulin sensitivity and sex on the phosphoproteome (Figure 2A).

Analysis of insulin-regulated phosphoproteome data using hierarchical clustering revealed 242 insulin-regulated phosphosites, with 186 phosphosites on 85 proteins showing increased phosphorylation upon insulin stimulation (class I) and 56 phosphosites on 33 proteins showing decreases by insulin stimulation (class II; Figure 2B and Supplemental Table 1). Within class I, we could identify 4 subclasses based on different phosphorylation patterns, as determined using a variable cut height approach (27). Class IA included 119 phosphosites that were equally upregulated by insulin stimulation in both I-Sen and I-Res men and women, as exemplified by $\mathrm{AKT}^{\mathrm{S473}}$ (where AKT1 indicates AKT serine/ threonine kinase 1) (Figure 2, B and C). Class IB represented 14 insulin-stimulated phosphosites that showed enhanced phosphorylation in the I-Res iMyos when compared with I-Sen iMyos, especially in cells from men, for example, STMN1 ${ }^{\mathrm{S} 25}$ (where STMN1 indicates stathmin) (Figure 2, B and C). Class IC included 33 insulin-stimulated phosphosites, which were hyperresponsive in I-Res myoblasts versus I-Sen myoblasts, with the effect being dominant in cells from women, e.g., AKT1S1 ${ }^{\mathrm{T} 246}$ (where AKT1S1 indicates proline-rich AKT1 substrate 1) (Figure 2, B and C). Finally, class ID represented 20 insulin-stimulated phosphosites that were hyperresponsive in the I-Sen iMyos, especially in women, as exemplified by NDRG2 ${ }^{\mathrm{S} 332}$ (where NDRG2 indicates NMYC downstream-regulated gene 2) (Figure 2, B and C).

Insulin downregulated phosphorylations (class II) could also be divided into 2 subcategories. Class IIA included 27 phosphosites that were approximately equally downregulated upon insulin stimulation in both I-Res men and women, for example, SH3BP2 ${ }^{\mathrm{S} 444}$ (where SH3BP2 indicates SH3 domain-binding protein 2) (Figure 2, B and C). Class IIB, on the other hand, was composed of 29 insulin-stimulated phosphosites that were downregulated by insulin in both I-Sen and I-Res myoblasts, but only in cells from women, e.g., SOWAHB ${ }^{\mathrm{S508}}$ (where SOWAHB indicates sosondowah ankyrin repeat domain family member B) (Figure 2, B and C). 
A

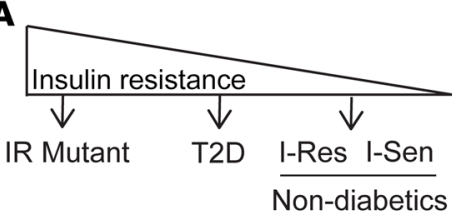

C
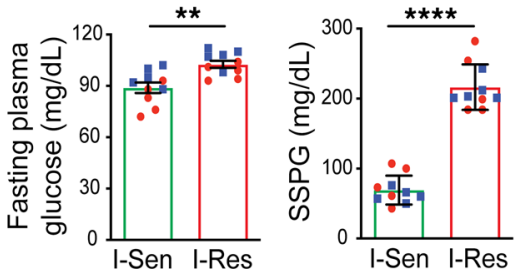
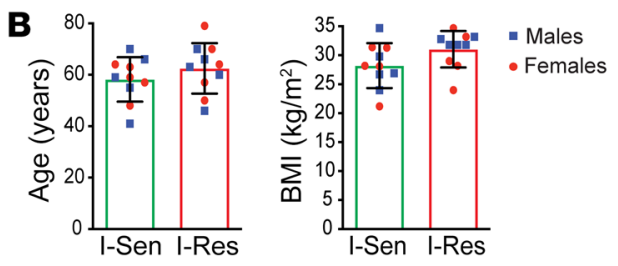

D Glucose uptake

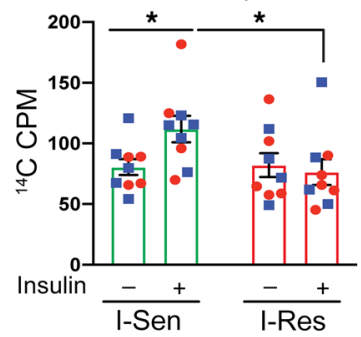

E

Males
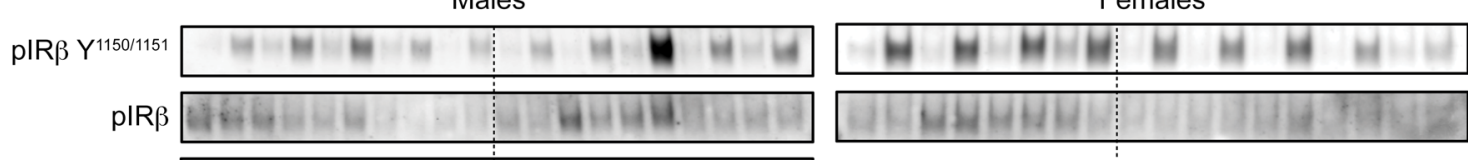

pAKT S ${ }^{473}$
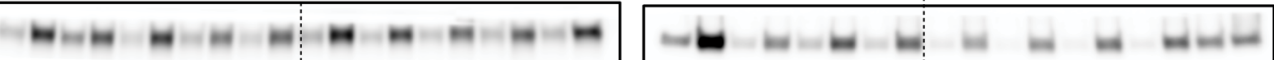

AKT E⿺辶⿻上丨)

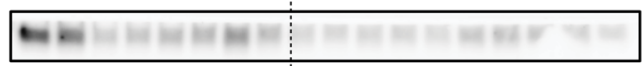

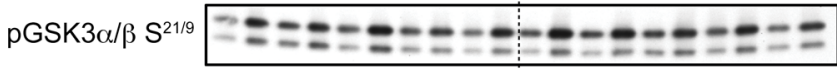

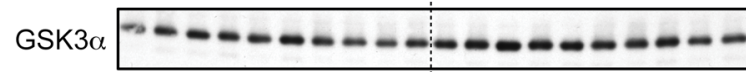

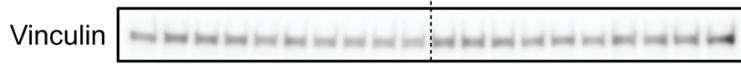

Insulin -+-+-+-+-+-+-+-+-+-+

I-Sen

I-Res

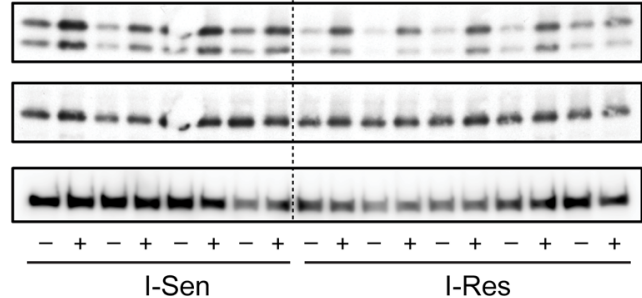

$\mathbf{F}$
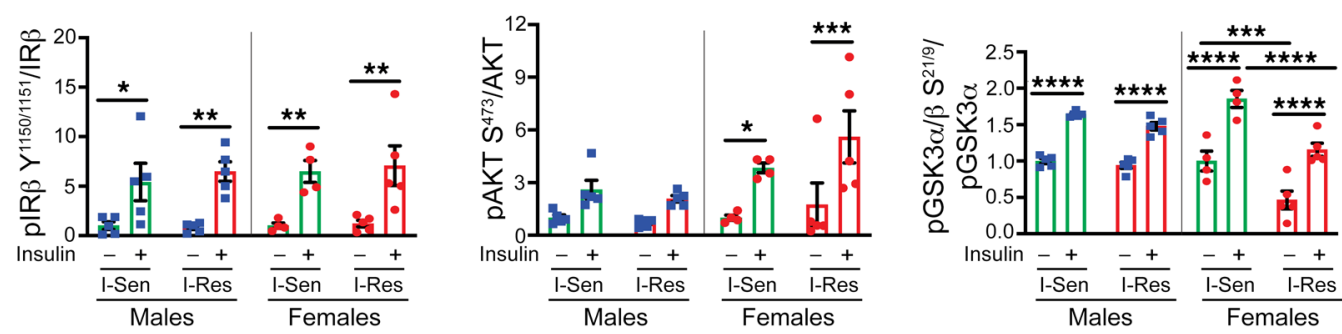

Figure 1. Metabolic characterization of I-Sen and I-Res iMyos. (A) Studies performed over the spectrum of insulin-resistance changes. (B) Age, BMI, (C) fasting plasma glucose, and SSPG data are shown for each I-Sen (green bars) and I-Res (red bars) subject (men, blue squares; women, red circles). Data are represented as mean \pm SEM. $n=10$ /group. ${ }^{* *} P<0.01 ;{ }^{* * *} P<0.0001$, unpaired $\mathrm{t}$ test. (D) 2-DOC glucose uptake assay in iMyos stimulated with $100 \mathrm{nM}$ insulin for 30 minutes. Data are represented as mean \pm SEM. $n=9 /$ group, ${ }^{*} P<0.05$, basal vs. insulin, and insulin I-Sen vs. I-Res, 2-way ANOVA followed by correction for multiple comparison by controlling the FDR. (E) Insulin-signaling Western blotting in I-Sen and I-Res iMyos from men and women showing phosphorylation of IR $\beta^{\text {V1150/1151 }}, \mathrm{AKT}^{5473}, \mathrm{CSK} 3 \alpha / \beta^{521 / 59}$, and their respective total protein levels. (F) Quantification of insulin-signaling experiments normalized by total protein expression. Data are represented as mean $\pm \mathrm{SEM} .{ }^{*} P<0.05 ;{ }^{* *} P<0.01 ;{ }^{* *} P<0.001 ;{ }^{* * *} P<0.0001$, basal vs. insulin and I-Sen vs. I-Res, 2-way ANOVA followed by correction for multiple comparison by controlling the FDR.

Thus, in classes I and II, i.e., the insulin up- and downregulated sites, about two-thirds of the sites were unaffected by the insulin-sensitivity status of the donor (classes IA and IIA), while about one-third were altered by differences in insulin sensitivity (classes IB, IC, ID, and IIB), with some of these changes seen in both men and women and others being present in cells from donors of only one sex. Reactome pathway analysis of the phosphosites in class I showed significant enrichment for pathways regulated by receptor tyrosine kinases (RTK) mTOR and ERK, while class II sites were enriched in pathways related to nuclear events, muscle contraction, and deubiquitination (Supplemental Figure 2C).

Integrated phosphoproteomic changes in nondiabetics and type 2 diabetics. In our previous study of iMyos from patients with insulin resistance due to T2D, we noted that in addition to the changes 
A
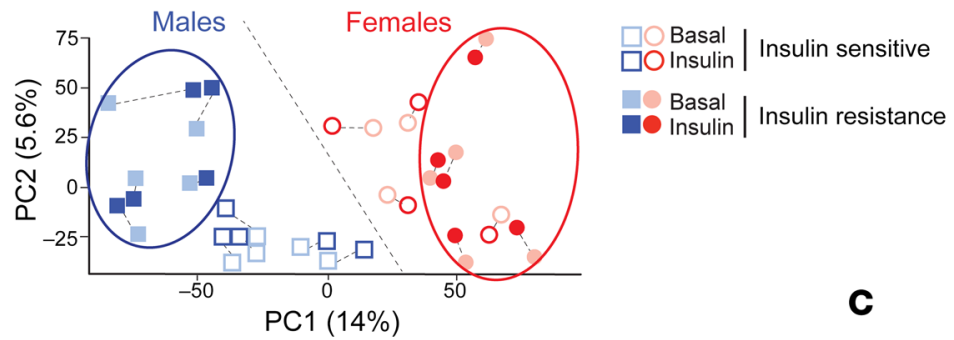

Class IA

Ins upregulated (119)

AKT1_S473

B

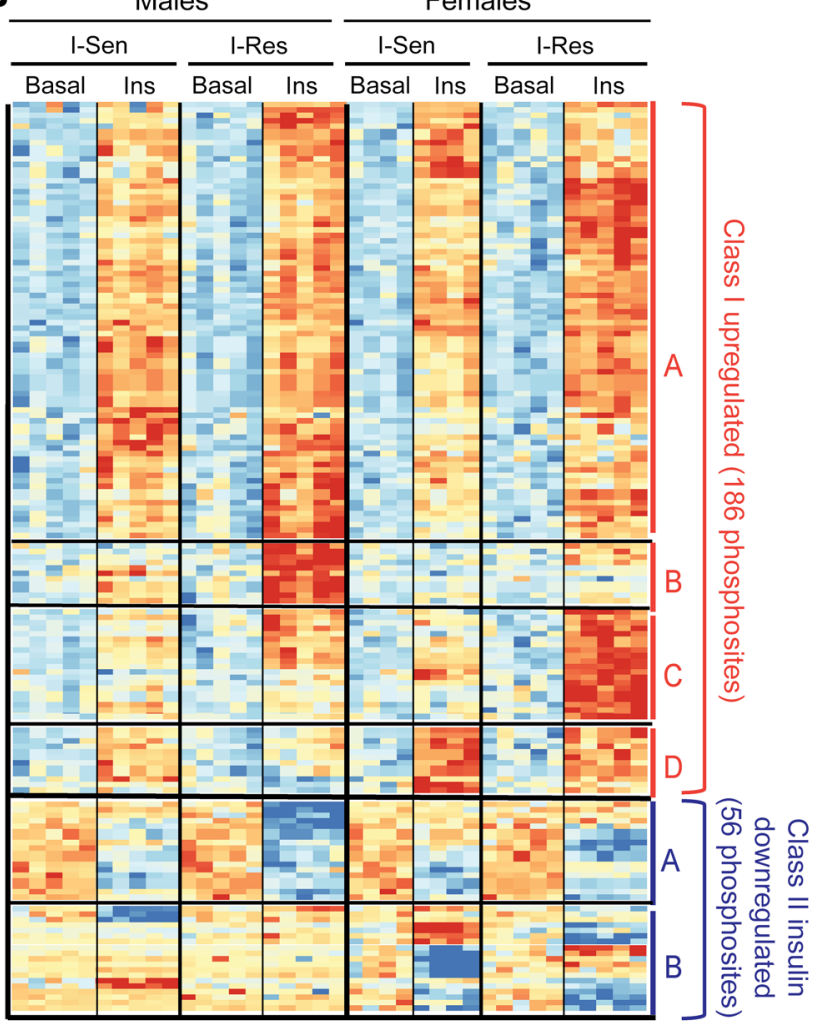

$\log _{2}$ intensity $Z$ score

$\begin{array}{lllll}-2 & -1 & 0 & 1 & 2\end{array}$

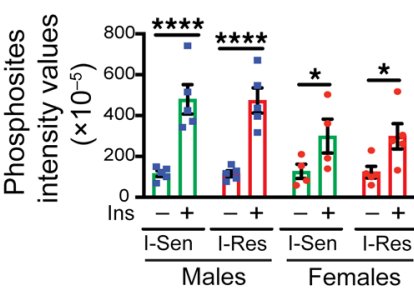

Class IC: Hyper-response in I-Res females (33)

AKT1S1_T246

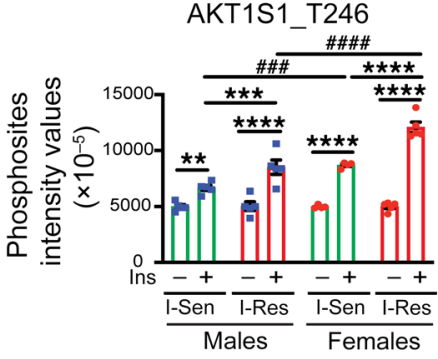

Class IIA: Ins downregulated in I-Res males and females (27) SH3BP2 S444

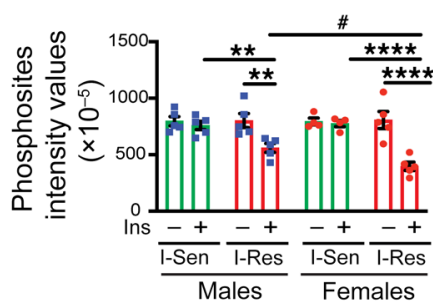

Class IB: Hyper-response in I-Res males (14)

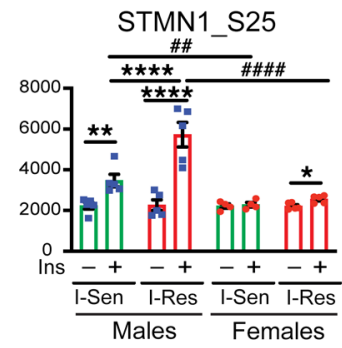

Class ID: Hyper-response in I-Sen females (20)

NDRG2 S332

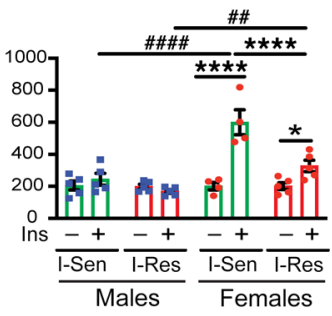

Class IIB: Ins downregulated in I-Sen \& I-Res females (29) SOWAHB S508

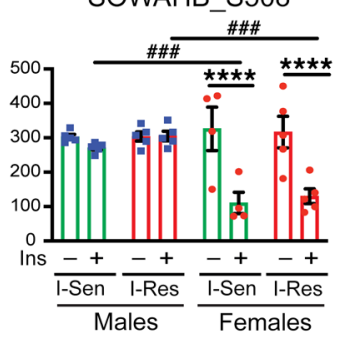

Figure 2. Overview of global phosphoproteome data and highlight of insulin-regulated changes. (A) PCA plot showing separation of the phosphoproteome data by subject sex (blue, men; red, women) and insulin-sensitivity status (open shape, insulin sensitive; filled shape, insulin resistant). A basal (lighter shade) and insulin-stimulated (darker shade) pair for each cell line is shown (dotted line connecting basal and stimulated pair). (B) Hierarchical clustering of the phosphopeptides showing insulin-regulated effects of insulin resistance. Rows represent $z$ scores of the log ${ }_{2}$-transformed intensity of phosphosites for each sample labeled in the column. (C) Quantification of representative phosphosites from each of the different clusters (classes IA-ID and IIA and IIB). Data are represented as mean \pm SEM of phosphosites intensity values $\left(\times 10^{-5}\right)$. ${ }^{*} P<0.05 ;{ }^{* *} P<0.01 ;{ }^{* *} P<0.001 ;{ }^{* * * *} P<0.0001$, basal

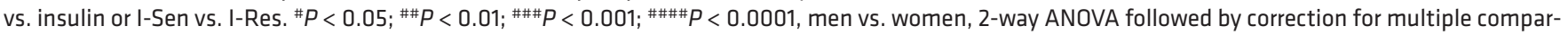
ison by controlling the FDR.

in insulin stimulation, there were also substantial changes in the levels of basal, i.e., non-insulin-stimulated, phosphorylation in iMyos from T2D versus control individuals. Indeed, this involved 732 phosphosites on 561 proteins (20). To better understand changes in phosphorylation-mediated signaling associated with insulin resistance, we sought to overlap the data from the current study of insulin resistance in the nondiabetic population with the previous study in T2D patients to identify common alterations in both the insulin-stimulation ratio for each of the phosphorylation events and the insulin-independent (basal) phosphorylation. Of a total of 7803 phosphosites identified in both studies, we found 389 phosphosites that showed significant changes in insulin-stimulation ratio shared in both nondiabetics and patients with T2D and an additional 197 sites that showed alterations in basal phosphorylation, independent of insulin stimulation, in both studies (FDR < 0.05; Figure 3A). Hierarchical clustering analysis of the 389 protein phosphorylation changes of the insulin-stimulation ratio revealed 214 phosphosites on 181 proteins whose ratio was 
upregulated in I-Res and T2D patients and 175 phosphosites on 129 proteins whose stimulation ratio was downregulated in I-Res and T2D patients (FDR $<0.05$; Figure 3B and Supplemental Table 2). The upregulated cluster was exemplified by CHAMP1 ${ }^{\mathrm{S} 173}$ (where CHAMP1 indicates chromosome alignment-maintaining phosphoprotein 1) and STMN1 ${ }^{\mathrm{S} 38}$, and the downregulated cluster, i.e., with significantly lower stimulation ratio in both I-Res and T2D patients, was exemplified by SRRM2 ${ }^{\mathrm{S} 876}$ (where SRRM2 indicates serine/arginine repetitive matrix protein 2) and $\mathrm{PHF}^{51722}$ (where PHF3 indicates PHD finger protein 3) (Figure 3C). Similarly, hierarchical clustering analysis of the 197 basal protein phosphorylation changes revealed 90 phosphosites on 70 proteins upregulated in cells from both I-Res nondiabetics and T2D patients and 107 phosphosites on 76 proteins downregulated in I-Res and T2D cells (FDR<0.05; Figure 3D and Supplemental Table 3). The phosphosites showing significantly higher phosphorylation in I-Res and $\mathrm{T} 2 \mathrm{D}$ patients are exemplified by $\mathrm{mTOR}^{\mathrm{S} 2481}$ and $\mathrm{LIMCH}^{\mathrm{S} 72}$ (where LIMCH1 indicates LIM and calponin homology domains-containing protein 1); those significantly downregulated in both I-Res and T2D independently of insulin stimulation are exemplified by $\mathrm{CDK}^{\mathrm{Y} 15}$ (where CDK1 indicates cyclin-dependent kinase 1) and SLC38A10 ${ }^{\mathrm{S} 82}$ (where SLC38A10 indicates putative sodiumcoupled neutral amino acid transporter 10) (Figure 3E).

An integrated signaling map showing the altered sites of phosphorylation that were present in iMyos from both I-Res nondiabetic and T2D patients is shown in Figure 4, and the biological process (Gene Ontology [GO]) analysis of these insulin-resistance changes is presented in Supplemental Figure 3. In the signaling map, basal upregulated and downregulated phosphosites identified in both studies are shown by purple and blue text, respectively, and the insulin-stimulated ratio upregulated and downregulated phosphosites are shown in red and green text, respectively (Figure 4).

At proximity to the IR, of the $8 \mathrm{Ser} / \mathrm{Thr}$ phosphosites identified on IRS-1 in both studies, only one showed consistent alteration in insulin resistance, and this was an upregulation of basal phosphorylation at IRS-1 Ser1101. Increased Ser1101 phosphorylation has been observed in cells after treatment by TNF- $\alpha$ (28) and in liver of obese mice (29). In addition, there was upregulation of basal phosphorylation of AKT Ser124 and mTOR Ser2481. On the other hand, insulin-regulated GSK3A Ser282 and basal TBC1D1 Ser237 sites were downregulated. Interestingly, insulin-regulated sites on FOXO3 and FOXK2 were upregulated, and phosphorylation of FoxK1 showed basal up- and downregulation on multiple sites, whereas neither of the FOXO1 phosphosites, Ser287 and Ser319, were consistently altered by insulin resistance.

Simultaneous analysis of insulin resistance in iMyos of both nondiabetics and T2D patients also identified a large network of insulin-regulated and basal protein phosphorylation changes related to outside the proximal insulin-signaling cascade. These included alterations in multiple regulators of Rab, Rac, and Rho GTPase signaling, including changes in phosphorylation of TBC1D10A and TBC1D10B, ARHGAP5 and ARHGAP12, and ARHGEF2, as well as many of their downstream targets involved in cytoskeleton organization. Phosphorylation of several proteins related to muscle contraction were also downregulated in the basal state. Even more striking, the phosphoproteomic analysis uncovered a signature of phosphorylation defects in various proteins in the nucleus. These included increased basal and insulin-regulated changes in phosphorylation of proteins related to gene expression. Similarly, phosphosites on proteins involved in DNA/chromatin organization and RNA splicing/processing showed both up- and downregulated phosphorylation changes in the basal and/or stimulated states. Taken together, these analyses demonstrate that insulin-resistance changes not only point to critical nodes of alteration in the classical insulin-signaling cascade, but also demonstrate a network of phosphorylation changes in proteins related to Rab/Rho/Rac signaling and actin remodeling/cytoskeleton organization as well as what we believe to be a novel nuclear signature in cells from both nondiabetics and diabetics ex vivo.

Sex-specific phosphoproteome fingerprint. PCA analysis of the phosphoproteomics data from nondiabetic individuals indicated that in addition to insulin resistance, the sex of the cell donor may be an important modulator of cell signaling. Indeed, hierarchical clustering analysis of insulin-independent protein phosphorylation, i.e., analysis of sites whose phosphorylation was not changed following insulin stimulation, revealed a striking 3784 phosphosites that differed significantly between cells from men and women (FDR < 0.05; Figure 5A and Supplemental Table 4), with 1965 phosphosites on 584 proteins showing significantly higher phosphorylation in cells from men as compared with cells from women (class III) and 1455 phosphosites on 682 proteins showing higher phosphorylation in cells from women compared with cells from men (class IV). There were 2 subclasses within each of these classes. Class IIIA was composed of phosphosites showing significantly higher phosphorylation in men than women that were not further altered by insulin resistance, as exemplified by CDC37513 (where CDC37 indicates Hsp90 cochaperone Cdc37) (Figure 5, A and B), whereas class IIIB consisted of phosphosites with greater phosphorylation in men than women that were also upregulated in the men by insulin resistance, for example, SRRM1 ${ }^{\text {T406 }}$ (where SRRM1 indicates serine and arginine repetitive matrix 1) (Figure 5, A and B). Conversely, class IVA represented phosphosites that were lower in men than women, for example, MARCKS ${ }^{5167}$ (where MARCKS indicates myristoylated alanine-rich C-kinase substrate) (Figure 5, A and B). Finally, class IVB included phosphosites that showed lower protein phosphorylation in men versus women, and this difference was magnified in insulin-resistant individuals, e.g., APAF1-interacting protein APIP ${ }^{887}$ (where APIP indicates methylthioribulose-1-phosphate dehydratase) (Figure 5, A and B). The sexual dimorphic patterns of phosphorylation of $\mathrm{CDC} 37^{\mathrm{s} 13}$ in class IIIA and MARCKS ${ }^{\mathrm{S} 167}$ in class IVA were confirmed by Western blotting using phosphosite-specific antibodies (Figure 5C).

Given the unexpected strong sexually dimorphic nature of changes in protein phosphorylation, we compared our current results to the phosphoproteomic changes in the T2D study, but focusing on only the iMyos from the nondiabetic subjects that served as controls (20). PCA of the combined data again demonstrated a clear separation of the phosphoproteome, with the sex of the cell donor being the strongest component in both study cohorts (Supplemental Figure 4A). Likewise, hierarchical clustering analysis revealed 902 phosphosites that differed significantly between cells from men and cells from women in both cohorts (FDR $<0.05)$, with 400 phosphosites showing significantly higher phosphorylation in women as compared with men and 502 phosphosites showing the 
A

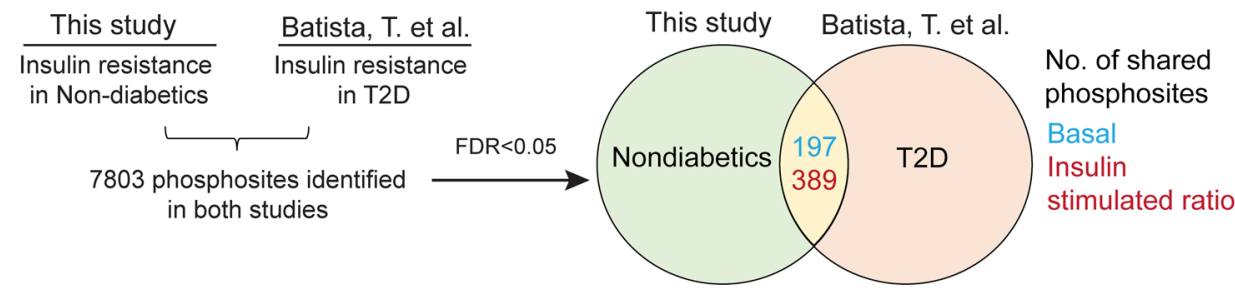

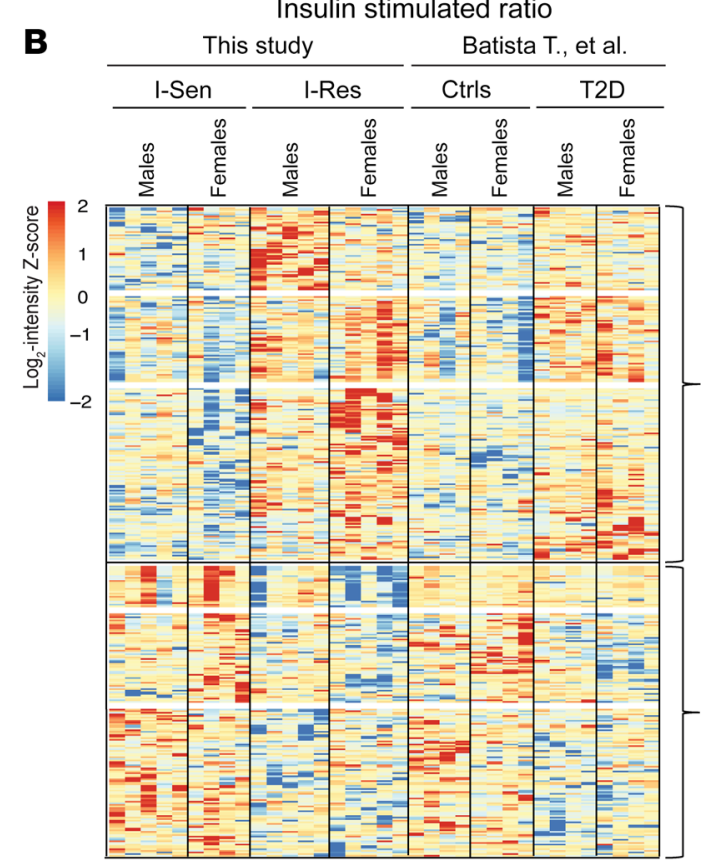

Insulin stimulated ratio
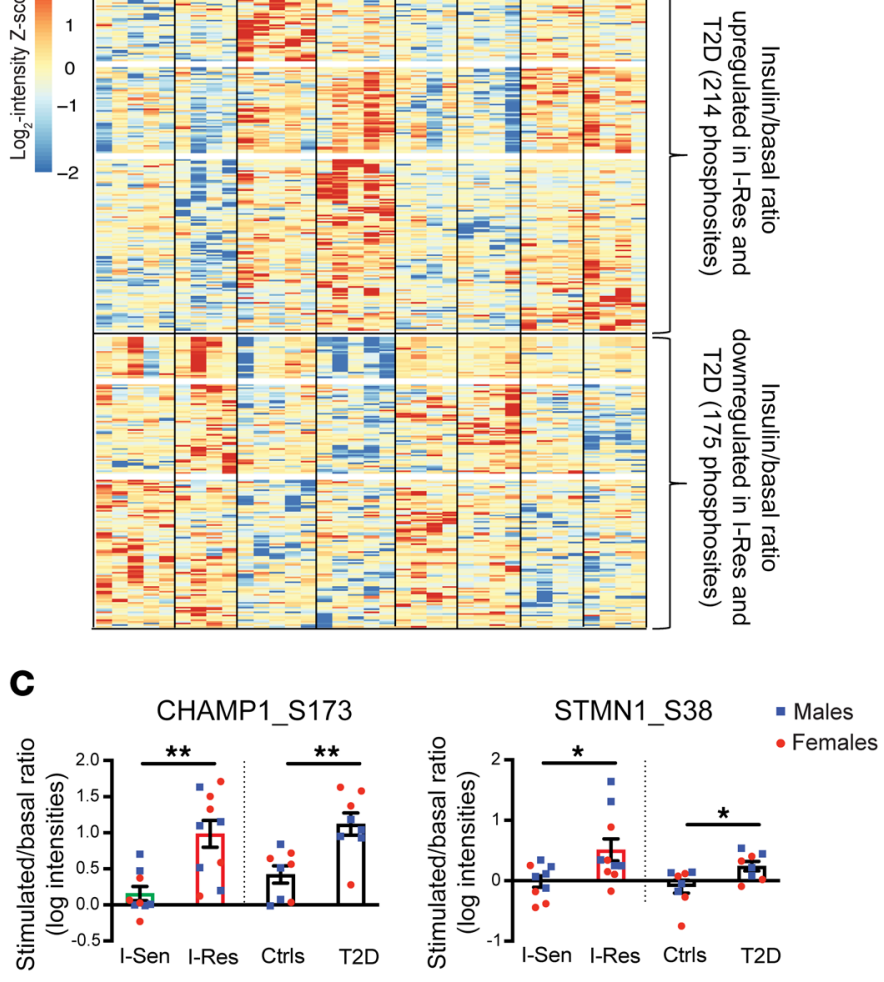

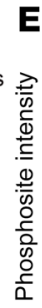
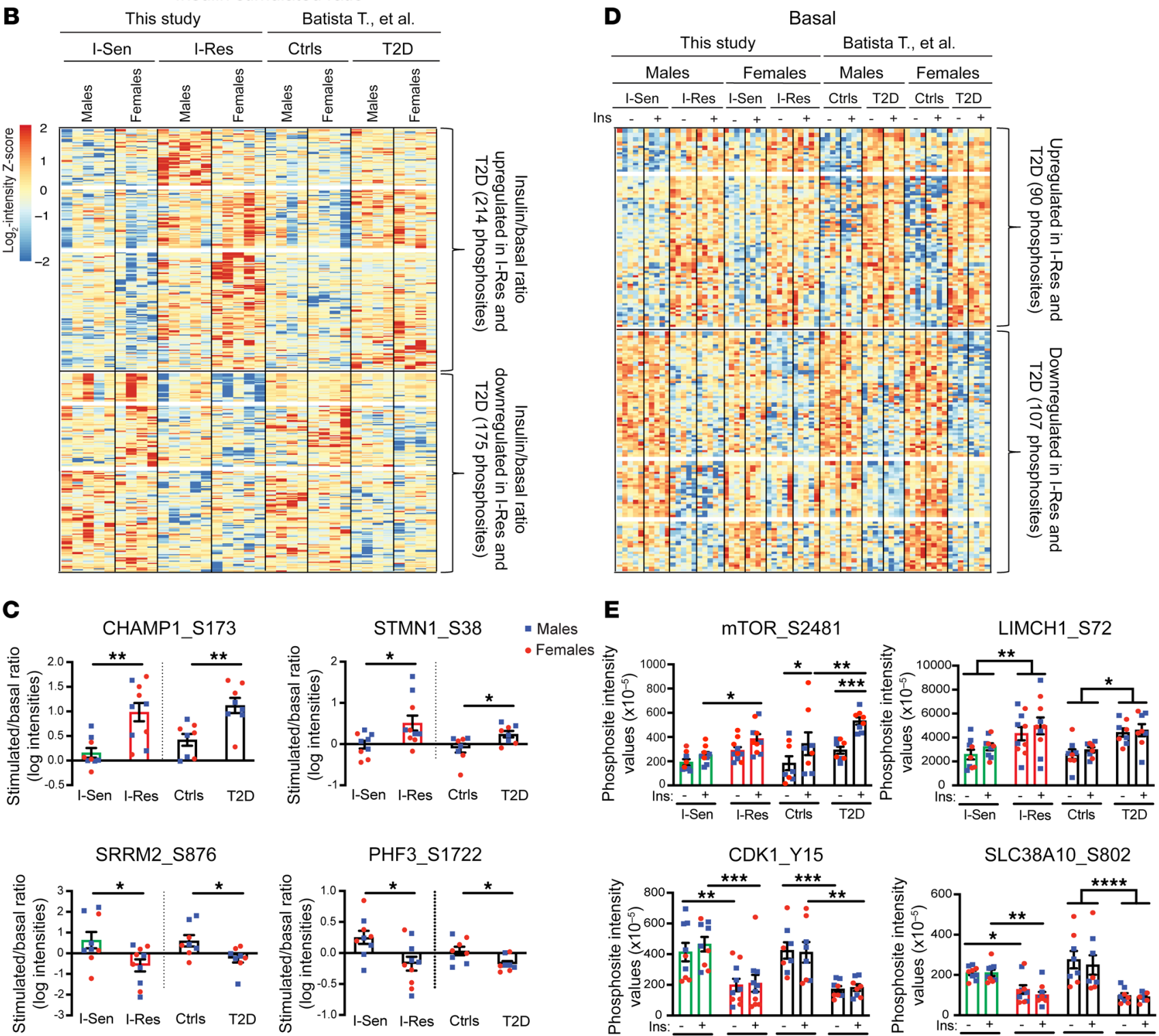

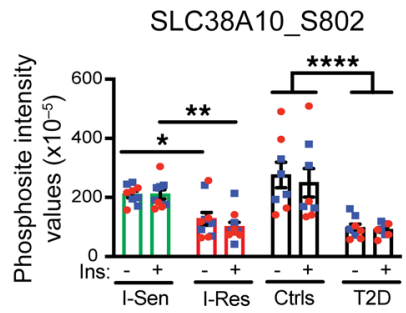

Figure 3. I-Res phosphoproteome changes shared with T2D. (A) Overview of the analysis to compare overlapping phosphoproteome changes in I-Res and T2D patients (FDR < 0.05). Data for Batista et al. can be found in ref. 20. (B) Hierarchical clustering of the insulin/basal ratio of the phosphopeptides showing shared phosphoproteome changes in I-Res and T2D iMyos. Rows represent $z$ scores of the $\log _{2}$-transformed intensity of phosphosites for each sample labeled in the column. (C) Quantification of representative phosphosites from each of the up- and downregulated clusters from B. Data are represented as mean \pm SEM of the phosphosites intensity values. ${ }^{*} P<0.05$; ${ }^{* *} P<0.01$, I-Sen vs. I-Res or T2D vs. controls, unpaired $t$ test. (D) Hierarchical clustering of the phosphopeptides showing shared phosphoproteome changes in I-Res and T2D iMyos at the basal state. Rows represent $z$ scores of the log ${ }_{2}$-transformed intensity of phosphosites for each sample labeled in the column. (E) Quantification of representative phosphosites from each of the up- and downregulated clusters from $\mathbf{D}$. Data for men and women were combined for clearer presentation. Data are represented as mean \pm SEM of the phosphosites intensity values. ${ }^{*} P<0.05$; ${ }^{* *} P<0.01$; ${ }^{* *} P<0.001$; ${ }^{* * *} P<0.0001$, basal vs. insulin or I-Sen vs. I-Res or T2D vs. controls, 2 -way ANOVA followed by correction for multiple comparison by controlling the FDR. 


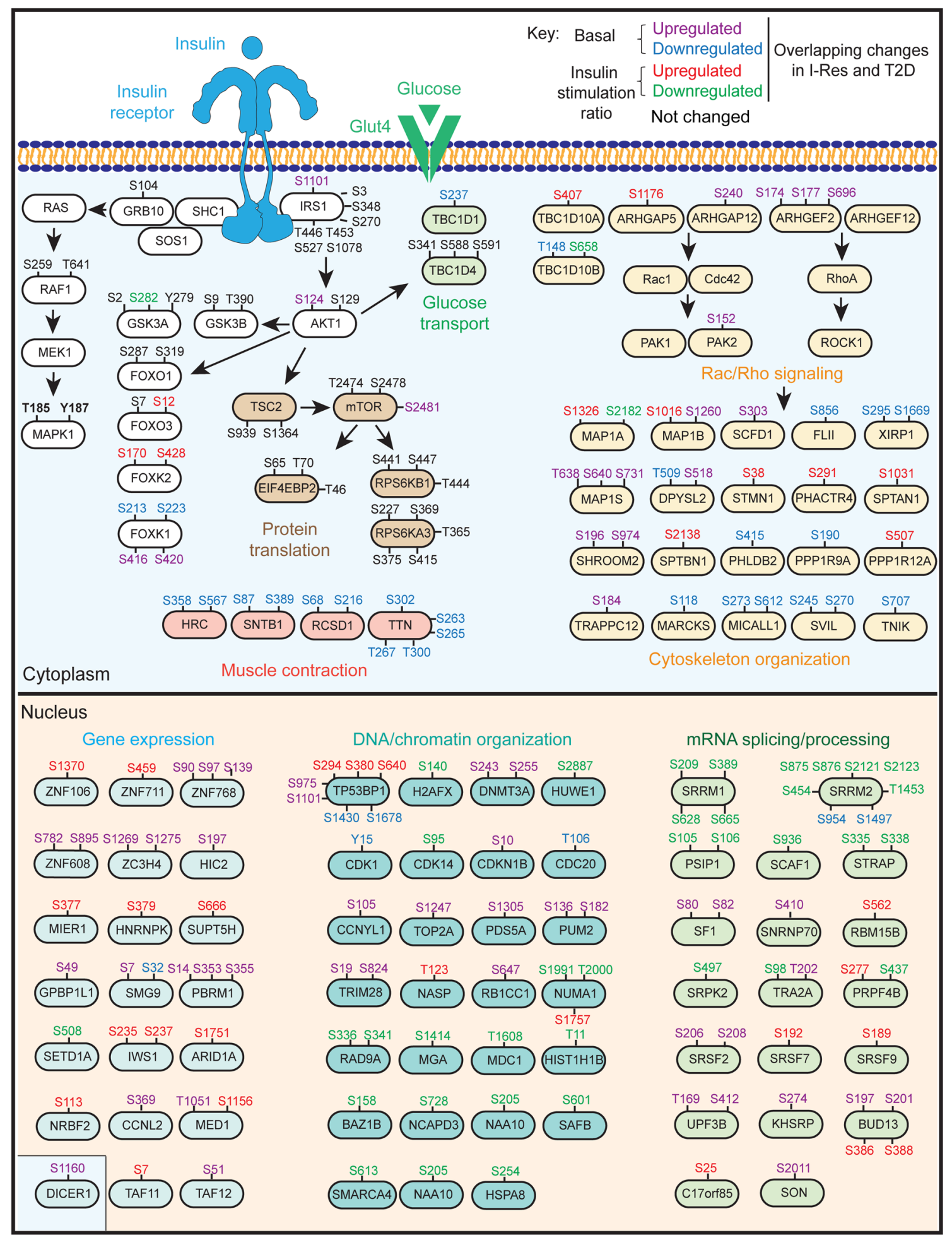

Figure 4. Signaling map highlighting critical nodes of phosphoproteome alterations overlapping in nondiabetics and T2D patients. Signaling map showing shared protein phosphorylation changes in the basal state (upregulated in purple, downregulated in blue) and insulin-stimulation ratio (upregulated in red, downregulated in green); those not changed are shown in black. The pathways shown are significantly enriched based on biological GO analysis (FDR $<0.05)$, and the related phosphosites show significant differences between I-Sen vs. I-Res and/or T2D vs. controls $(P<0.05)$. The map was drawn using Adobe Illustrator 2020. 
A

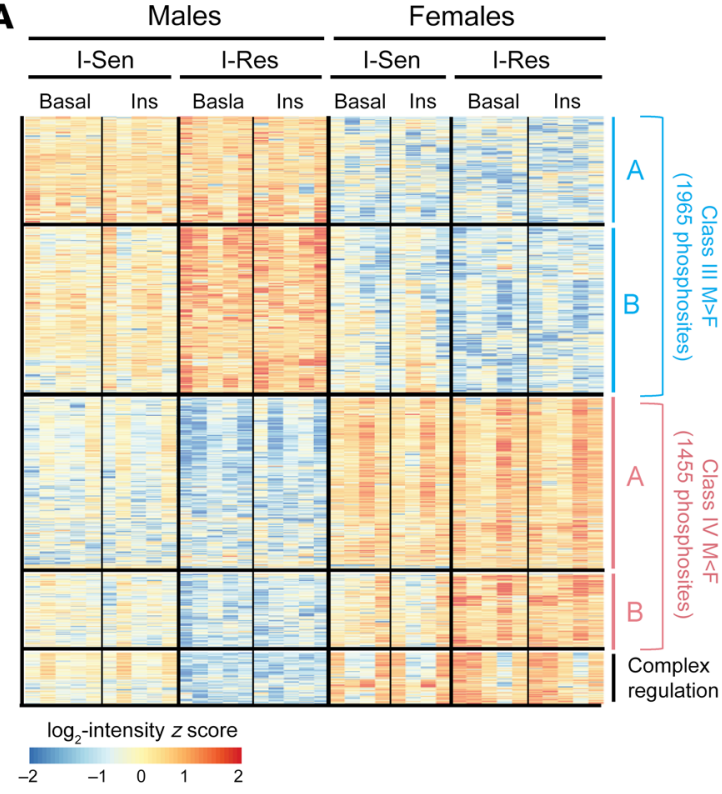

C $\frac{\text { Males }}{\text { I-Sen I-Res }} \frac{\text { Females }}{\text { I-Sen I-Res }}$

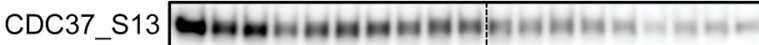
MARCKS_S167

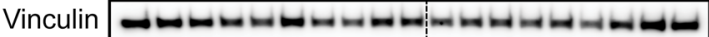

B

sexual dimorphism

CDC37_S13

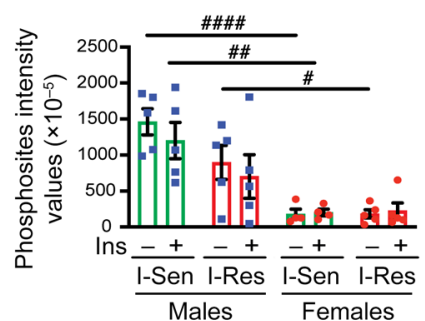

Class IVA: sexual dimorphism \& I-Res downregulated in males

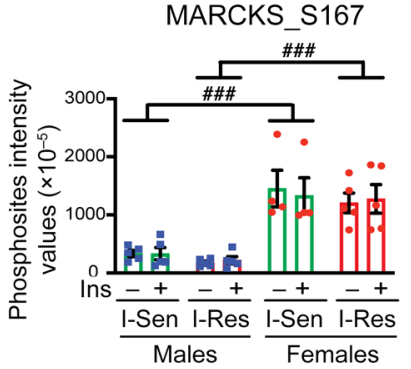

Class IIB: sexual dimorphism

\& I-Res upregualted in males SRRM1_T406

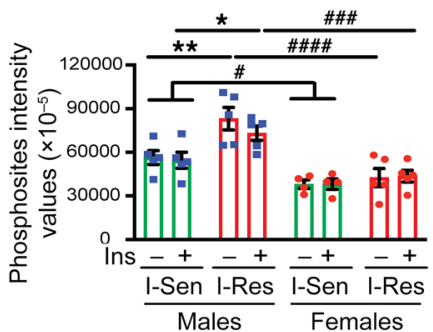

Class IVB: sexual dimorphism \& I-Res upregulated in females

APIP_S87

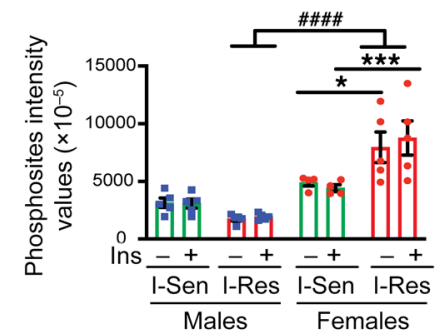

D

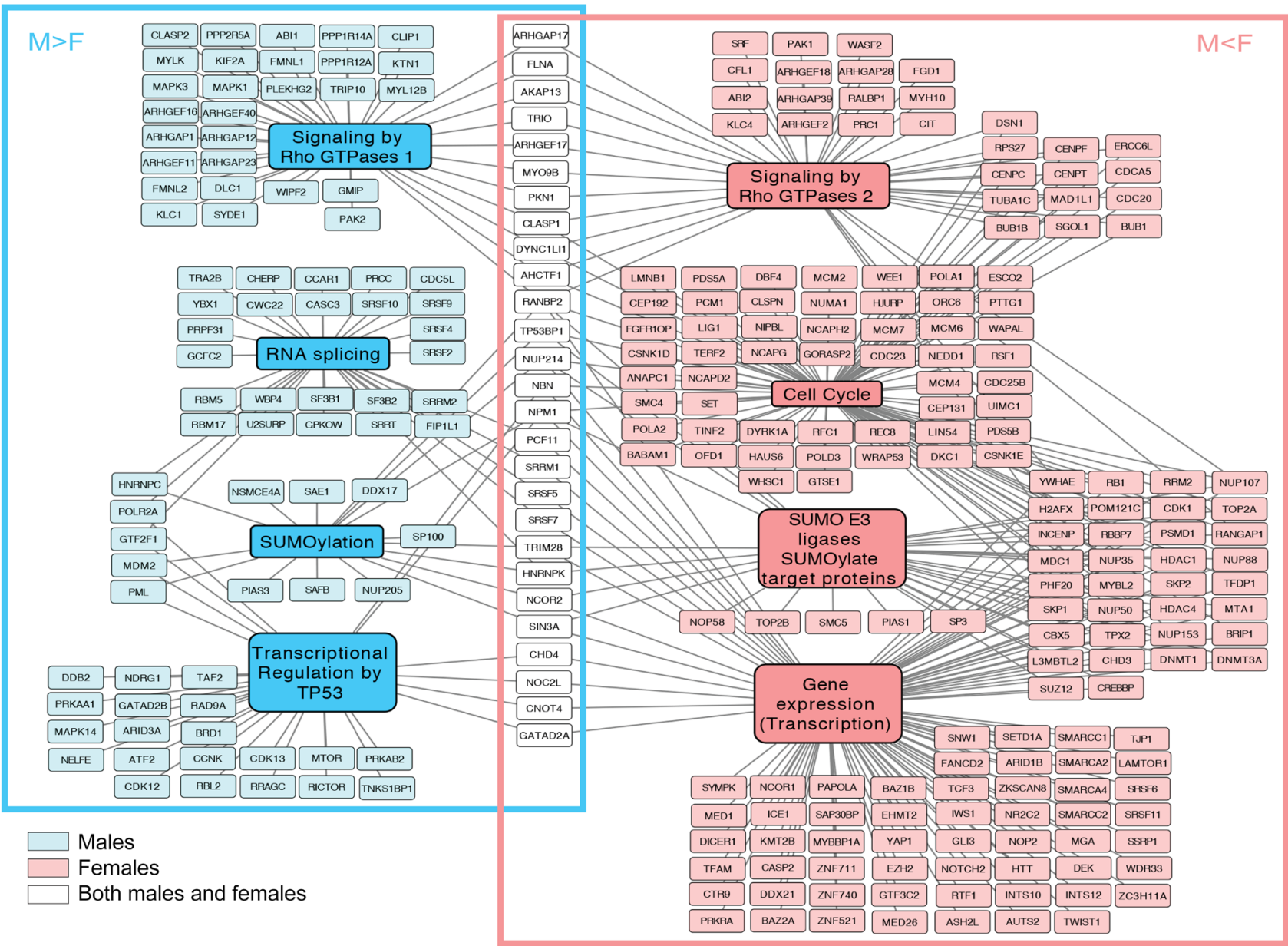


Figure 5. Insulin-independent protein phosphorylation alterations highlighting the sexual dimorphism. (A) Hierarchical clustering of phosphopeptides showing sexual dimorphism and insulin-resistance changes. Rows represent $z$ scores of the $\log _{2}$-transformed intensity of phosphosites for each sample labeled in the column. (B) Quantification of representative phosphosites from each of the different clusters (classes IIIA and IIIB and classes IVA and IVB). Data are represented as mean \pm SEM of phosphosites intensity values $\left(\times 10^{-5}\right) .{ }^{*} P<0.05 ;{ }^{* *} P<0.01 ;{ }^{* *} P<0.001$, I-Sen vs. I-Res. ${ }^{\# P}<0.05$; ${ }^{\#} P<0.0$; ${ }^{\# \# P}>0.001$; $\# \# \# P<0.0001$, men vs. women, 2 -way ANOVA, followed by correction for multiple comparison by controlling the FDR. (C) Validation of a few phosphosites by immunoblotting in I-Res and I-Sen iMyos from men and women at the insulin-stimulated state. (D) Map representation of the most enriched biological pathways and related proteins in men (blue) and women (pink). The map is drawn using Cytoscape software (version 3.8.0) and Adobe Illustrator 2020.

opposite pattern (Supplemental Figure 4, B and C). Thus, in addition to any differences associated with insulin-sensitivity status, protein phosphorylation exhibits unique patterns specific to men and women, even in vitro in the absence of added sex hormones.

Reactome pathway analysis revealed a small number of highly linked biological pathways underlying the sexual dimorphism in phosphoproteomic changes (Supplemental Figure 5A and Figure 5D). For example, cells from both men and women showed sex-specific differences in phosphorylation of proteins involved in Rho GTPase signaling, but these occurred largely on different proteins in this pathway depending on sex. Likewise, men dominated over women in phosphorylation of proteins involved in mRNA processing/splicing, while women had higher phosphorylation of proteins involved in the cell cycle and DNA metabolism. Significant sex differences were also observed in proteins involved in SUMOylation versus SUMO E3 ligases and ubiquitination and in transcriptional regulation by TP53 versus overall changes in transcription and gene expression (Figure 5D). As a result, even though similar pathways were regulated in cells of both men and women, the protein phosphorylation pattern showed sex-specific differences, with only a small fraction of the proteins in these pathways showing equal phosphorylation in cells from men and cells from women (Figure 5D).

To identify potential upstream drivers of these sexually dimorphic phosphorylation differences, we performed kinase-substrate enrichment analysis (Supplemental Figure 5B). Phosphosites in class IIIA, which showed higher levels of phosphorylation in cells from men, were potential targets of the casein kinases (CK2A2, CK2A) and the serine/threonine kinase ATR, whereas members of the MAP kinase family (ERK1/2, P9ORSK, MEK1, mTOR, ROCK1) were potential upstream drivers of class IIIB phosphorylations, i.e., sites higher in men than women and upregulated in by insulin resistance (Supplemental Figure 5B). On the other hand, sites of upregulation of protein phosphorylation in cells from women in classes IVA and IVB were predicted targets for the cyclin-dependent kinases (CDK and CDC2) and CAMK2 and MARK2 kinases, respectively (Supplemental Figure 5B). Again, it is important to emphasize that the sexual dimorphic differences in protein phosphorylation occurred in vitro and in the absence of sex hormones.

Functional implications of the phosphoproteome. Given the striking effects of insulin resistance and sex on the phosphoproteome, we assessed potential functional implications in two important pathways: the DNA damage/checkpoint pathway and the Rho GTPase pathway. DNA damage has been implicated in diabetes complications (30) and can be assessed by the number of apurinic/apyrimidinic (AP) sites, i.e., sites that have neither a purine nor a pyrimidine base, in DNA (31). An important driver of the DNA damage response is the protein tumor suppressor p53-binding protein 1 (TP53BP1), and this protein showed higher basal phosphorylation of Ser1430 in women versus men and a significant reduction in phosphorylation specifically in iMyos from I-Res men (Figure $6 \mathrm{~A})$. This reduction in phosphorylation correlated with a significantly reduced number of AP sites in DNA in the cells of I-Res men, but not women, indicative of differences in DNA damage repair in these cells (Figure 6B). The Rho GTPase pathway, on the other hand, is involved in the cytoskeleton remodeling required for normal regulation of glucose metabolism (32). As noted above, there was altered phosphorylation of both ARHGAPs and ARHGEFs, which differed based on both sex and insulin-resistance status. Likewise, basal phosphorylation on Thr230 and Ser2 of PAK1, a Ser/Thr kinase linked to RhoA, Cdc42 and Rac1 activation, showed distinct and opposite differences in phosphorylation in cells of men and women, with a significant, more than $50 \%$, reduction in PAK1 ${ }^{\mathrm{T} 230}$ phosphorylation in cells from I-Res men. Cells from I-Res women, in contrast, showed a trend to increased PAK phosphorylation at this site, whereas the PAK2 ${ }^{\mathrm{S} 2}$ site was highly male dominant (Figure 6C). RhoA activation assessed in these same iMyos using a pull-down strategy showed differences in stoichiometry, which paralleled these phosphorylation differences. Thus, iMyos from women showed lower levels of active RhoA, which increased in the insulin-resistant cohort, while iMyos from men showed higher levels of RhoA activation, which decreased in the insulin-resistant cohort (Figure 6, D and E). Taken together, these data demonstrate how the phosphoproteomic changes are reflected in functional differences in the DNA damage response and actin cytoskeleton remodeling and are affected by both insulin-resistance status and sex of the donor.

\section{Discussion}

Insulin resistance is a complex metabolic phenotype and is central to pathophysiology of a variety of diseases, including obesity, $\mathrm{T} 2 \mathrm{D}$, and metabolic syndrome. In diseases such as diabetes, insulin resistance can be driven by a variety of circulating extrinsic factors that are altered in disease pathogenesis, including free fatty acids, ceramides, cytokines, adipokines, and even circulating exosomal miRNAs, which have been shown to alter both upstream and downstream aspects of insulin signaling (reviewed in ref. 33). Recently, we have shown that iPSCs from T2D patients differentiated into myoblasts in vitro demonstrate insulin resistance and a large network of altered signaling, indicating T2D-intrinsic or cell-autonomous defects (20). Consistent with this, insulin resistance can be identified in offspring of T2D parents many years prior to disease (4). Insulin resistance is also present in a significant fraction of the general population, where it has been shown to predispose to metabolic syndrome (7). In the present study, we have explored the cell-autonomous determinants of insulin resistance in the general population using iMyos generated from nondiabetic individuals over the range of insulin sensitivity. Our data show that these cells exhibit large differences in their phosphoproteome 
A

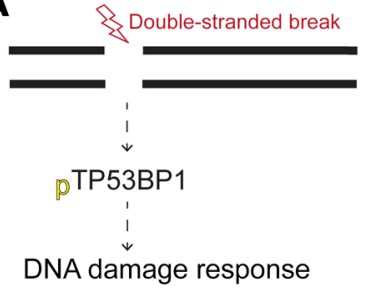

TP53BP1_S1430

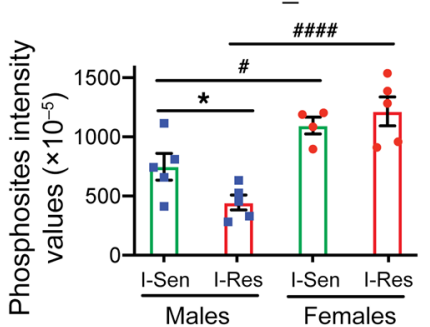

B

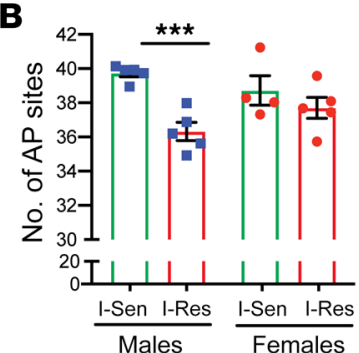

C
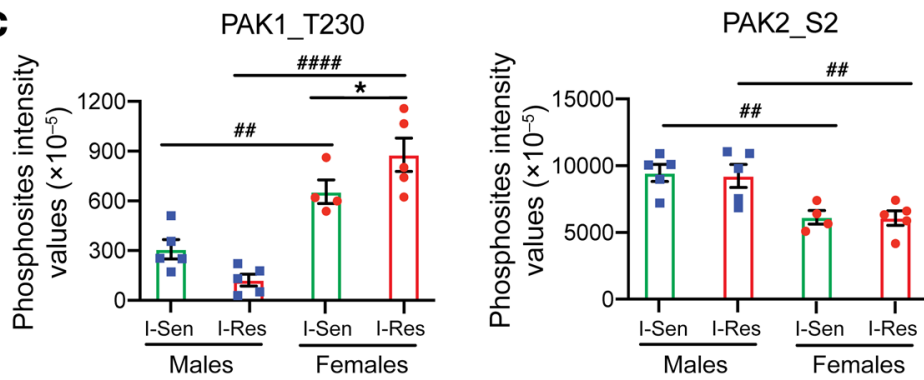

D

Females Males

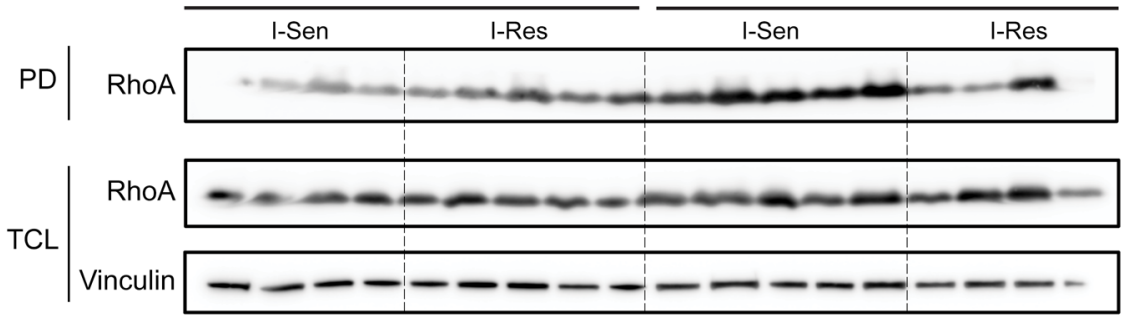

E

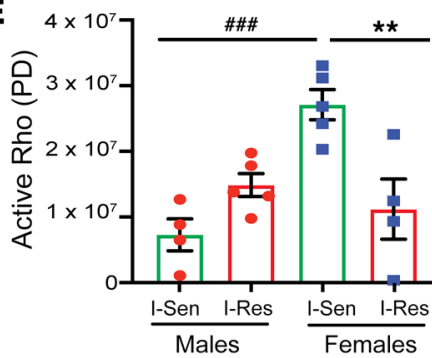

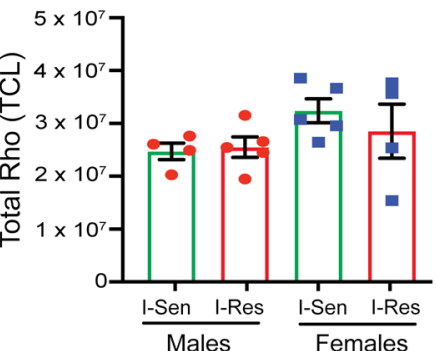

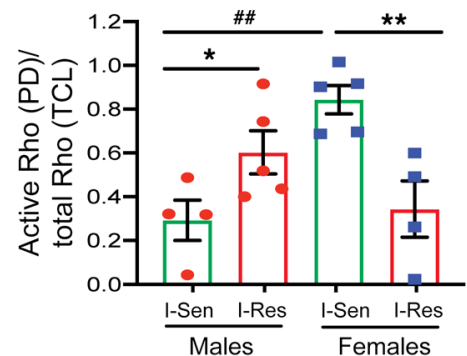

Figure 6. Functional implication of the phosphoproteomics alterations. (A) DNA damage response overview and quantification of TP53BP1 phosphosite. Data are represented as mean \pm SEM of the basal phosphosites intensity values $\left(\times 10^{-5}\right)$. ${ }^{*} P<0.05$, I-Sen vs. I-Res. ${ }^{\#} P<0.05$; \#\#\#\# $P<0.0001$, men vs. women, 1-way ANOVA followed by correction for multiple comparison by controlling the FDR. (B) Quantification of the number of AP sites for equal amounts of each of the DNA samples from iMyos as per manufacturer's instructions for ELISA. Data are represented as mean \pm SEM. ${ }^{* * *} P<0.001, \mathrm{I}-\mathrm{Sen}$ vs. I-Res, 1-way ANOVA followed by correction for multiple comparison by controlling the FDR. (C) Quantification of PAK1 and PAK2 phosphosites. Data are represented as mean \pm SEM of the basal phosphosites intensity values $\left(\times 10^{-5}\right)$. ${ }^{*} P<0.05$, I-Sen vs. I-Res. ${ }^{\# \#} P<0.01$; \#\#\# $P<0.0001$, men vs. women, 1-way ANOVA, followed by correction for multiple comparison by controlling the FDR. (D) Western blot of the I-Sen and I-Res iMyos from cell lysates from women and men processed through the active RhoA pull-down (PD) experiment and the total cell lysates (TCL). (E) Quantification of the raw pull-down Western blot showing active form of RhoA as well as total cell lysates showing total RhoA levels. Vinculin is used as a loading control. Data are represented as mean \pm SEM. ${ }^{*} P<0.05$; ${ }^{*} P<0.01$, I-Sen vs. I-Res. ${ }^{* \#} P<0.01$; \#\# $P<0.001$, men vs. women, 1-way ANOVA, followed by correction for multiple comparison by controlling the FDR.

based on insulin-resistance status and that many of these overlap with the alterations observed in cells from T2D patients, thus highlighting key steps through which to target insulin resistance. In addition, we find that the sex of the cell donor further modifies intracellular signaling and that these changes can be reflected in differences in downstream biological responses in these cells.

Changes in phosphoproteome related to insulin resistance reveal several important categories of proteins. In the proximal insulin signaling cascade, we find increased basal phosphorylation of IRS-1 Ser1101 in cells from both nondiabetic and diabetic individuals. This site has been previously shown to be increased in phosphorylation in insulin resistance in obese mice (29) and after treatment of cells by TNF- $\alpha$ (28) and has been ascribed to be a target of PKC $\theta$ (28), IKK, and S6 kinase (29). Increased serine/ threonine phosphorylation of IRS-1 has been shown to modulate stability and tyrosine phosphorylation of the protein, thus creating 
feedback regulation in both physiological and pathological states (reviewed in ref. 34). Treatment of cells with L-citrulline reduces phosphorylation of Ser1101 in IRS-1 and is associated with improved insulin signaling (35).

Another consistent finding in insulin resistance in the cells from nondiabetics and T2D patients is increased phosphorylation of AKT1 at Ser124 and mTOR at Ser2481. Phosphorylation of AKT1 at Ser124 has previously been shown to regulate the extent of AKT activation independently of PI3K $(36,37)$. This site has also been shown to be phosphorylated by $\mathrm{PKC} \zeta$, as well as regulated by the atypical PKC-interaction protein Par-4, leading to reduced Akt enzymatic activity (38). Ser2481 in mTOR is a site of autophosphorylation and is associated with activation of mTORC (39), a kinase that has many downstream targets affecting protein translation. In addition to its physiological roles, mTOR has been postulated to play an important role in the development of insulin resistance via its activation by branched chain amino acids (40) and obesity (reviewed in ref. 41). Another important alteration is decreased phosphorylation of TBC1D1 at Ser237 in both I-Res and T2D iMyos. This site has been shown to be induced with exercise and to correlate with the activity of AMPK and thus is normally positively associated with glucose transport (42).

In addition to changes in the canonical insulin-signaling cascade, there are many alterations in signaling that are conserved in cells from nondiabetic insulin-resistant individuals and cells from $\mathrm{T} 2 \mathrm{D}$ patients. These occurred in pathways involved in regulation of Rab/Rho/Rac GTPase signaling, actin/cytoskeletal organization, regulation of chromatin structure, gene expression, and RNA processing and splicing. Among transcriptional regulators, it is interesting to note that while no alterations in FOXO1 phosphorylation were observed in both populations of cells, there were consistent changes in one site on FOXO3 (Ser12) and multiple sites in FOXK1 and FOXK2 phosphorylations. FOXK1 showed downregulated basal phosphorylation of Ser213,223 and upregulated phosphorylation of Ser416,420, i.e., changes in the opposite direction of the phosphorylations induced by insulin action (17). These sites are thought to be target sites of GSK3 and mTOR and have been shown to affect the translocation of FOXK1 in and out of the nucleus $(17,43)$. Also of note are the family of ZNF transcription factors, such as ZNF106, ZNF608, ZNF711, and ZNF768, along with various other proteins involved in regulation of gene expression, whose phosphorylation was upregulated in basal and insulin-stimulated states in nondiabetic insulin-resistant and T2D iMyos.

Another interesting set of proteins whose phosphorylation was altered by insulin resistance were proteins involved in mRNA splicing and processing. Previous studies in rodents have shown that insulin regulates the expression of several genes encoding proteins involved in constitutive and alternative mRNA splicing in muscle (44). Altered spliceosome function due to downregulation of the splicing factor SFRS10 has also been observed in liver and muscle biopsies of obese humans (45). The altered phosphorylation network also included multiple proteins involved in chromatic organization and DNA repair. Clinical studies have provided evidence that pathophysiological alterations in diabetes, such as hyperglycemia, hyperinsulinemia, advanced glycation end products, and free fatty acids, can contribute to DNA damage, including DNA strand break and base oxidation (46). In addition, we observed changes in RhoA activation based on insulin-sensitivity status and sex of the individual. It remains to be determined how alterations in the activity of the Rho signaling pathway might contribute to insulin resistance in a sex-dependent manner in iMyos. Overall, this comprehensive map of the overlapping changes in nondiabetics and T2D patients in our study demonstrates a large network of altered critical nodes associated with insulin resistance.

In addition to the insulin resistance-related changes, the most striking finding of this study is the unexpected differences in protein phosphorylation in cells from men versus women. Indeed, we identified a network of 3420 phosphosites in 1143 unique proteins, which exhibited significantly differential phosphorylation based on sex, with almost 2000 phosphosites showing higher phosphorylation in iMyos from men versus women and over 1450 sites that showed the opposite pattern (FDR < 0.05). Like the insulin-resistance changes, these were confirmed in a second independent cohort of cell lines from control individuals (20). Importantly, these sex-dependent differences in phosphorylation occurred in vitro in a setting in which cells from men and women were in identical tissue culture media and without the addition of sex hormones, thus showing cell-autonomous sex-specific phosphorylation changes. Most of the proteins involved in these differences were in a limited number of complementary pathways. Thus, cells from men showed enhanced phosphorylation of proteins associated with transcription, Rho GTPases, SUMOylation, mRNA splicing, and membrane trafficking, while proteins whose phosphorylation showed female dominance included those involved in cell cycle, chromatin organization, gene expression, Rho GTPase cycle, and protein ubiquitination. To our knowledge, such sexual dimorphism in signaling has not been previously reported, although recently, Oliva et al. identified sex differences in the transcriptome and its genetic regulation across a range of human tissues in vivo (47).

We also found an interaction between the sex effects and the insulin-resistance effects, which could be observed at the level of phosphorylation, as well in functional downstream readouts of these pathways. In the US, men have a slightly higher prevalence of diabetes compared with women (14.0\% vs. $12.0 \%$; ref. 48 ), despite having a significantly lower prevalence of obesity (49). Consistent with this, women have higher insulin-stimulated glucose uptake in skeletal muscle in vivo as compared with men, despite lower lean mass (50). Also, weight loss yielded greater reduction in risk for diabetes in men than in women in the participants of the diabetes prevention program (DPP) (51), and sex was identified as one of the significant predictors of the progression of nondiabetic offspring of parents with T2D into prediabetes in the pathobiology of prediabetes in a biracial cohort (POP-ABC) study (52). Furthermore, impaired fasting glucose is more prevalent in men than women in some populations, whereas impaired glucose tolerance is more pronounced in women than men (53). Independently of diabetes, it also is well known that the risk of development of coronary heart disease is much greater in men than in premenopausal women (54), although this difference disappears after menopause, suggesting that it is due to differences in hormonal milieu rather than cell-autonomous differences between men and women. Sex-specific differences have also been observed in the risk of stroke related to $\mathrm{T} 2 \mathrm{D}$, with the relative risk being higher in women than in men (55). Therefore, even though the prevalence of diabe- 
tes is only modestly skewed toward men versus women, the progression from normal glycemia to prediabetes as well as metabolic alterations linked to insulin resistance and skeletal muscle physiology can have sexually dimorphic phenotypes.

The sexually dimorphic protein phosphorylation pattern observed in our study may be linked to several potential upstream regulators. Although only approximately $4 \%$ of the proteins involved in differential phosphorylation between men and women are encoded on the $\mathrm{X}$ chromosome, the $\mathrm{X}$ chromosome encodes several protein kinases that might play a role in some of these protein phosphorylation differences either directly or secondarily by regulation of autosomally encoded protein kinases or phosphatases. Indeed, in the study noted above exploring sex differences in gene expression using databases containing information on 44 human tissues, $37 \%$ of all genes had sex-biased expression changes in at least one tissue (47). Finally, sex-hormone-dependent epigenetic modification could also play a role in exerting maleand female-specific phosphoproteome signature, although iPSC reprogramming is known to erase most epigenetic marks (56). Another point to be noted is that the BMI of both the I-Sen and I-Res individuals fell within the overweight to slight obese range. Whether this might exert any effect on insulin signaling via epigenetic imprinting remains to be determined; however, as noted above, iPSC reprograming erases most epigenetic marks.

In summary, phosphoproteomic analysis of human iMyos demonstrates a large network of dysregulated phosphorylations linked to differences in insulin resistance and insulin sensitivity as well as the sex of the cell donor. An important fraction of these is shared with cells from patients with T2D, indicating a more primal role in cell-intrinsic, possibly genetically programmed, insulin resistance. These include altered phosphorylation of IRS1 Ser1101, AKT Ser124, mTOR Ser2481, and TBC1D1 Ser237, all phosphorylations that can negatively influence insulin sensitivity. In addition, insulin resistance is marked by altered phosphorylation in a wide array of processes involving DNA repair, mRNA processing, cellular organization, protein translation, and cellular signaling outside the insulin action pathway. These latter alterations are further modified by the sex of the donor. For at least two of these pathways, DNA repair and RhoA activation, we find functional differences in cellular response that parallel the changes in upstream signaling. Importantly, all of these differences in the phosphoproteome related to sex and state of insulin sensitivity are retained in cells studied in vitro and after genetic reprogramming, indicating the cell-autonomous or intrinsic nature of these defects. Further studies will be needed to identify which kinases/phosphatases link the different pathways in this network and how this sexually dimorphic nature of the phosphoproteome affects normal physiology and the risk of different metabolic diseases between men and women. Nonetheless, these findings point to important critical nodes in insulin resistance that can serve as sites for future therapeutic development.

\section{Methods}

\section{Study subjects, SSPG, and iPSC reprogramming}

The iPSC lines used in this study were generated from 20 study subjects who had been recruited and assessed for insulin sensitivity using
SSPG obtained from the modified insulin-suppression test (57) at the Stanford University, School of Medicine Clinical and Translation Research Unit as part of the NIH-sponsored GENESiPS project. Briefly, the SSPG method involves an overnight fast, followed by simultaneous infusion of octreotide, insulin, and glucose at fixed doses, followed by blood collections at 10-minute intervals from 150 to 180 minutes after the infusion. This combined infusion allows glucose and insulin to both be in steady state, leading to the SSPG.

iPSC lines were generated as described previously (24), and those used in the study were chosen from 10 in the upper quintile of insulin sensitivity (I-Sen) and 10 in the lowest quintile of insulin sensitivity (I-Res), matched for age, sex, and race/ethnicity based on the SSPG (24).

\section{iPSC culture and myogenic differentiation}

The iPSCs were cultured on plates coated with hESC-qualified Matrigel (Corning) using the mTeSR1 media containing the $5 \mathrm{X}$ complement (StemCell Technologies) and passaged as aggregates using ReLeSR (StemCell Technologies). For differentiation into myoblasts, a modified version of the 2-step differentiation protocol was used based on a previous study (25). First, approximately $7 \times 10^{3} \mathrm{iPSCs} / \mathrm{cm}^{2}$ were seeded onto collagen I-coated plates (BioCoat, Fisher) in skeletal muscle cell growth basal medium (Lonza) containing $5 \%$ horse serum (HS), $50 \mu \mathrm{g} / \mathrm{mL}$ fetuin, $3 \mu \mathrm{M}$ CHIR99021, $2 \mu \mathrm{M}$ Alk5 inhibitor, $1 \mathrm{ng} / \mathrm{mL}$ bFGF, $10 \mathrm{ng} / \mathrm{mL}$ human recombinant EGF (hr-EGF), $10 \mu \mathrm{g} / \mathrm{mL}$ insulin, $0.4 \mu \mathrm{g} / \mathrm{mL}$ dexamethasone, $10 \mu \mathrm{M}$ Y27632 (ROCK inhibitor), and $200 \mu \mathrm{M}$ ascorbic acid with media change every 2 days, which resulted in myogenic precursor/satellite-like (SC-like) cells within 10 days. The SC-like cells were then trypsinized and plated at approximately $7 \times 10^{3}$ iPSCs $/ \mathrm{cm}^{2}$ onto collagen I-coated plates (BioCoat, Fisher) in skeletal muscle cell growth basal medium (Lonza) containing 5\% HS, 50 $\mu \mathrm{g} / \mathrm{mL}$ fetuin, $10 \mu \mathrm{g} / \mathrm{mL}$ insulin, $0.4 \mu \mathrm{g} / \mathrm{mL}$ dexamethasone, $10 \mu \mathrm{M}$ Y27632 (ROCK inhibitor), $10 \mathrm{ng} / \mathrm{mL}$ hr-EGF, $20 \mathrm{ng} / \mathrm{mL}$ hr-hepatocyte growth factor (HGF), $10 \mathrm{ng} / \mathrm{mL}$ hr-PDGF AB, $10 \mathrm{ng} / \mathrm{mL}$ oncostatin $\mathrm{M}$, $20 \mathrm{ng} / \mathrm{mL}$ bFGF, $10 \mathrm{ng} / \mathrm{mL}$ IGF1, $2 \mu \mathrm{M}$ SB431542, and $200 \mu \mathrm{M}$ ascorbic acid with media change every 2 days. This resulted in myoblasts (iMyos) within another 10 days.

\section{DNA, RNA isolation, and qPCR}

DNA was isolated using DNAzol (Thermo Fisher), and the samples were processed further for the AP site ELISA assay as described below. Total RNA from all the cell types was isolated using TRIzol (Thermo Fisher) following the chloroform/isopropanol/ethanol extraction method. cDNA was synthesized from 400 ng of RNA using a High Capacity cDNA Reverse Transcription Kit (Applied Biosystems), and the resulting cDNA was used for the quantitative PCR (qPCR) reaction with iQ SYBR Green Supermix (Bio-Rad, catalog 1708884) performed on a C1000 Thermal Cycler (Bio-Rad, catalog CFX384). TATA box-binding protein (Tbp) was used as housekeeping gene to normalize gene expression. Primer sequences used were as follows: Tbp (forward: TGATGCCTTATGGCACTGGACTGA, reverse: CTGCTGCCTTTGTTGCTCTTCCAA), Nanog (forward: TCCAACATCCTGAACCTCAG, reverse: GACTGGATGTTCTGGGTCTG), Sox2 (forward: GCCGAGTGGAAACTTTTGTCG, reverse: GGCAGCGTGTACTTATCCTTCT), DNMT3B (forward: ATAAGTCGAAGGTGCGTCGT, reverse: GGCAACATCTGAAGCCATTT), POU5F1/Oct4 (forward: GTGGAGGAAGCTGACAACAA, reverse:CAGGTTTTCTTTCCCTAGCT), Pax7 (forward: CGTGCTCAGAATCAAGTTCG, reverse: GTCAGGTTC- 
CGACTCCACAT), Pax3 (forward: AGAAGGCCAAACACAGCATC, reverse: TTCTGCGCTGTTTCCTCTTT), MyoG (forward: AGATGTGTCTGTGGCCTTCC, reverse: AGCTGGCTTCCTAGCATCAG), and MyoD1 (forward: CTCCAACTGCTCCGACGGCAT, reverse: ACAGGCAGTCTAGGCTCGACAC).

\section{Insulin signaling, protein extraction, immunoblotting, and immunostaining}

For insulin stimulation, the iMyos were washed twice with PBS and incubated with starvation media containing Ham's F10 and 0.1\% BSA for 4 to 6 hours before stimulation with $100 \mathrm{nM}$ insulin for $10 \mathrm{~min}$ utes. Cells were washed twice with ice-cold PBS and lysed using RIPA buffer supplemented with protease and phosphatase inhibitor. Equal amounts of proteins $(\sim 10 \mu \mathrm{g})$ were resolved by SDS-PAGE and transferred to PVDF membrane (Bio-Rad). Following incubation in PBS containing $0.01 \%$ Tween-20 (PBS-T) and $10 \%$ dry milk or $5 \%$ BSA, membranes were probed with the following antibodies: $p I R \beta^{\mathrm{Y} 1150 / 1151}$ (catalog 3024, Cell Signaling Technology), IR $\beta$ (catalog sc-711, Santa Cruz Biotechnology Inc.), AKT S473 (catalog 9271, Cell Signaling Technology), AKT (catalog 4685, Cell Signaling Technology), p-GSK3 $\alpha$ S21/ßS9 (catalog 8566, Cell Signaling Technology), GSK3a (catalog 9338, Cell Signaling Technology), CDC37 S13 (catalog 13248, Cell Signaling Technology), MARCKS S167 (catalog 8722, Cell Signaling Technology), and vinculin (catalog MAB3574, Millipore Sigma). Membranes were then incubated with appropriate HRP-conjugated secondary antibodies. Signal was detected by chemiluminescence using the ChemiDoc Touch Imaging System (Bio-Rad) and quantified with ImageLab software (version 6.0.1, build 34) (Bio-Rad).

For immunofluorescence analysis of MyoD1, iMyos grown on 24-well plates were fixed with $4 \%$ paraformaldehyde, permeabilized with $0.1 \%$ Triton-X, blocked with $10 \%$ normal donkey serum, and incubated with the MyoD1 primary antibody (catalog 13812, Cell Signaling Technology) overnight at $4^{\circ} \mathrm{C}$. The following day, cells were washed and incubated with Alexa Fluoro 488 donkey anti-rabbit secondary antibody for 1 to 2 hours at room temperature. The cells were also stained with DAPI (1:1000, Thermo Fisher) for 10 minutes at room temperature and then imaged using an Olympus IX51 inverted fluorescence microscope.

\section{Clucose uptake, quantification of AP sites in DNA, and active Rho pull-down assays}

For the glucose uptake assay, iMyos grown in a 96-well plate were serum starved (DMEM F/12 + 0.25\% BSA) overnight, washed, and incubated with Krebs-Ringer bicarbonate HEPEs (KRBH) buffer $\left(120 \mathrm{mM} \mathrm{NaCl}, 10 \mathrm{mM} \mathrm{NaHCO}, 4 \mathrm{mM} \mathrm{KH}_{2} \mathrm{PO}_{4}, 1 \mathrm{mM} \mathrm{MgSO}{ }_{4}\right.$, $1 \mathrm{mM} \mathrm{CaCl}_{2}, 30 \mathrm{mM} \mathrm{HEPES}$ ) for 1 hour at $37^{\circ} \mathrm{C}$, then stimulated with 100 $\mathrm{nM}$ insulin for 30 minutes. Following insulin stimulation, $13 \mu \mathrm{L}$ reaction mixture containing $0.1 \mu \mathrm{Ci}$ 2-DOG (PerkinElmer) and $200 \mu \mathrm{M}$ nonradiolabeled 2-DOG (MilliporeSigma) was added to each well and incubated for 10 minutes at $37^{\circ} \mathrm{C}$. Glucose uptake reaction was stopped by the addition of $26 \mu \mathrm{L}$ of $200 \mathrm{mM}$ nonradiolabeled 2-DOG and immediate transfer of the plates to an ice bath. Cells were washed 3 times with PBS and lysed with $100 \mu \mathrm{L}$ RIPA buffer, followed by scintillation counting.

For the functional assays, including the quantification of the number of AP sites in DNA through ELISA (STA-324, Cell BioLabs) and active RhoA pull-down assay (8820, Cell Signaling Technology), differentiated iMyos were processed according to the manufacturers' protocols.

\section{Statistics}

Data analysis was performed using appropriate unpaired or paired 2-tailed Student's $t$ test (GraphPad Prism software, version 8.4.3), and $P<0.05$ was considered to be significant.

\section{Phosphoproteomic analysis of iMyos}

Lysis and digestion. The basal and insulin-stimulated iMyos were processed as previously described (58). In brief, cells were washed with ice-cold PBS, lysed in SDC digestion buffer containing 4\% SDC, 100 $\mathrm{mM}$ Tris $\mathrm{pH} 8.5$, and were snap frozen. The samples were boiled, sonicated for 20 cycles in Biorupter Plus (Diagenode) and vortexed; protein concentration was determined by BCA assay. For each sample, $750 \mu \mathrm{g}$ of protein was alkylated with $10 \mathrm{mM} \mathrm{CAA}$ and reduced with $40 \mathrm{mM}$ TCEP by incubating for 20 minutes on ice in the dark. Then the samples were mixed with LysC and Trypsin (1:100 ratio) proteases and incubated overnight at $37^{\circ} \mathrm{C}$, mixed at $161 \mathrm{~g}$, in ThermoMixer.

Phosphopeptide enrichment. The resulting digested peptides were further mixed in the ThermoMixer for 30 seconds at $252 \mathrm{~g}$ along with $750 \mu \mathrm{l} \mathrm{ACN}$ and $250 \mu \mathrm{lK}$ buffer (36\% TFA and $3 \mathrm{mM} \mathrm{KH}_{2} \mathrm{PO}_{4}$ ). Any debris was cleared by centrifugation at $18,928 g$ for 15 minutes, and the supernatant was transferred to a $2 \mathrm{ml}$ Deepwell Plate (Eppendorf). For the phosphopeptide enrichment step, $\mathrm{TiO}_{2}$ beads (prepared in $80 \%$ ACN, 6\% TFA buffer) were added (1:10 ratio protein/beads) and incubated at $40^{\circ} \mathrm{C}, 448 \mathrm{~g}$, for 5 minutes in the ThermoMixer. The TiO2 bound phosphopeptides were pelleted by centrifugation, transferred to clear tubes, and washed 4 times using a wash buffer containing $60 \%$ ACN and 1\% TFA to remove nonspecific or nonphosphorylated peptides. The beads were suspended in a transfer buffer (80\% ACN, 0.5\% acetic acid), then transferred on top of single-layer C8 StageTips (stop and go extraction tips) and centrifuged until dryness. The phosphopeptides were obtained with elution buffer (40\% ACN, 20\% $\left.\mathrm{NH}_{4} \mathrm{OH}\right)$ and concentrated in a SpeedVac for 20 minutes at $45^{\circ} \mathrm{C}$. This was followed by phosphopeptides acidification by the addition of $100 \mu \mathrm{L}$ of $1 \%$ TFA. The acidified peptides were loaded onto equilibrated styrene divinylbenzene-reversed phase sulfonated (SDBRPS) StageTips for desalting and further cleanup. These SDBRPS StageTips were washed once in isopropanol/1\% TFA and twice with $0.2 \%$ TFA. Finally, the desalted phosphopeptides were eluted with $60 \mu \mathrm{L}$ elution buffer $(80 \%, 1.25 \%$ $\mathrm{NH}_{4} \mathrm{OH}$ ). The dried elutes were resuspended in MS loading buffer (3\% ACN, $0.3 \% \mathrm{TFA}$ ) and stored at $-20^{\circ} \mathrm{C}$ until MS measurement.

LC-MS/MS measurement. The phosphopeptides were analyzed using Q Exactive HF-X Hybrid Quadrupole-Orbitrap Mass Spectrometer (Thermo Fisher Scientific) coupled to a nanoflow EASY-nLC1000 HPLC (Thermo Fisher Scientific). The phosphopeptides were loaded onto a $50 \mathrm{~cm}$ C18 column with a $75 \mu \mathrm{M}$ inner diameter with the temperature maintained at $50^{\circ} \mathrm{C}$ by an in-house made column oven. The phosphopeptides were separated over a duration of 140 minutes using a mobile phase system buffer A (0.1\% formic acid) and buffer B (60\% ACN plus $0.1 \%$ formic acid) at a flow rate of $300 \mathrm{~mL} / \mathrm{min}$. Furthermore, the electrosprayed peptides were analyzed by the Q Exactive HF-X Hybrid Quadrupole-Orbitrap Mass Spectrometer (Thermo Fisher Scientific) in a data-dependent mode, with 1 survey scan at a target of $3 \times 10^{6}$ ions (300-1650 m/z, R=60,000 at $200 \mathrm{~m} / \mathrm{z}$ ), followed by Top10 MS/MS scans with high-energy collisional dissociation-based (HCD-based) fragmentation (target $1 \times 105$ ions, maximum filling time $120 \mathrm{~ms}$, isolation window $1.6 \mathrm{~m} / \mathrm{z}$, and normalized collision energy $27 \%)$ detected in the Orbitrap $(R=15,000$ at $200 \mathrm{~m} / \mathrm{z})$. Apex trig- 
ger (4-7 s), charge exclusion (unassigned, $1,5,-8$, and >8), and dynamic exclusion of $40 \mathrm{~s}$ were enabled.

Phosphoproteomics data analysis. The acquired raw data files were processed using the MaxQuant (59) software environment (version 1.5.5.2) with the built-in Andromeda search engine for identification and quantification of phosphopeptides. The data were searched using a target-decoy approach with a reverse database against UniProt Human (August 2016 version) reference proteome fasta file with an FDR of less than $1 \%$ at the level of proteins, peptides, and modifications. A few minor changes to the default settings used were as follows: oxidized methionine (M), acetylation (protein N-term), and in the case of phosphopetide search, phospho (STY) were selected as variable modifications and carbamidomethyl (C) as a fixed modification. A maximum of 2 missed cleavages was allowed; a minimum peptide length of 7 amino acids and enzyme specificity was set to Trypsin. In addition, the run algorithm was enabled. The MaxQuant output phospho (STY) table was processed using the Perseus (ref. 60; version 1.5.2.11) software suite. Prior to the analysis, residues marked as potential contaminants and reverse hits were filtered out. Phosphopeptides that had more than $80 \%$ valid values in at least one group were selected for downstream analysis. Missing values were replaced by random numbers that were drawn from normal distributions with means that were 1.6 times sample SDs downshifted from the sample means and SDs that were 0.6 times the sample deviations. Values were $\log _{2}$ transformed and further normalized to make all samples have the same median. The statistical significance of phosphopeptides was assessed with empirical Bayesian linear modeling using the limma package with default priors (61). $P$ values were corrected using the Benjamini-Hochberg FDR. Hierarchical cluster analysis was performed using a variable cut height approach based on the Euclidean distance of the significant phosphopeptides (27). Clusters were defined according to the hierarchical tree. The human kinase substrates analysis was performed using PhosphositePlus (62) and RegPhos (63) and was tested using Fisher's exact test.

Comparison of phosphoproteome data from 2 independent studies was performed by adjusting for the batch effect using surrogate variable analysis (64). Furthermore, the overlapping sites in nondiabetic and T2D iMyos were then identified through comparison of differential phosphosites in the control groups of each study (I-Sen and controls) and case groups of each study (I-Res and T2D) using a single combined data file containing both I-Res and T2D sets of the phosphoproteomics raw data. The phosphoproteomics data set generated for this study was deposited in the PRoteomics IDEntifications (PRIDE) database (PXD022637).
All phosphosites were determined using MaxQuant software. For those proteins for which the identified phosphosite was in a specific isoform, we adjusted the amino acid numbering system shown in Figure 4 to match the major isoform identified in UniPort. These included GSK3A S219, Y216, TBC1D4 S749, ARHGEF2 S695, PHACTR4 S448, and SF1 S205, and S207, which are now designated as GSK3A S282, Y279, TBC1D4 S588, ARHGEF2 S696, PHACTR4 S291, SF1S 80, and S82 respectively.

\section{Study approval}

All study subjects provided informed consent at the time of sample collection, which occurred at the Stanford University, School of Medicine.

\section{Author contributions}

$\mathrm{NH}$ designed and performed all experiments, analyzed all data, designed the figures, and wrote the paper. JL designed the study and prepared samples for phosphoproteomics. AKJ designed the study and analyzed and performed the phosphoproteomics. Cofirst authorship was assigned based on the listed author contributions. TMB contributed to experiments and critical reading of the paper. HP and JMD performed bioinformatics analysis. ICO and JWK provided the I-Sen and I-Res iPSC lines and contributed to the study design and critical reading of the paper. MM supervised the phosphoproteomics work. CRK conceived the study, helped with data analysis and interpretation, reviewed and edited the manuscript, and supervised the project. All authors read, reviewed, and edited the manuscript.

\section{Acknowledgments}

We thank Leslie Caron from the University of Sydney for sharing the protocol and technical expertise for the differentiation of iPSCs into myoblasts. We also thank Igor Paron and Christian Deiml for their support in the mass spectrometry analysis (MPI Biochemistry). AKJ and MM were supported by the Max Planck Society for the Advancement of Science and by the German Research Foundation (DFG/Gottfried Wilhelm Leibniz Prize). This work was supported by NIH grants R01DK031036, R01DK033201 (to CRK); P3ODK036836 (to the Joslin Diabetes Center); R01DK116750, R01DK120565, R01DK106236, R01DK107437, and P30DK116074 (to JWK); and ADA 1-19-JDF108 (to JWK); and the Mary K. Iacocca Professorship (to CRK).

Address correspondence to: C. Ronald Kahn, Joslin Diabetes Center, One Joslin Place, Boston, Massachusetts 02215, USA. Phone: 617.309.2635; Email: c.ronald.kahn@joslin.harvard.edu.
1. Ferrannini E, et al. Hyperinsulinaemia: the key feature of a cardiovascular and metabolic syndrome. Diabetologia. 1991;34(6):416-422.

2. Haffner SM, et al. Prospective analysis of the insulin-resistance syndrome (syndrome X). Diabetes. 1992;41(6):715-722.

3. Kuk JL, Ardern CI. Age and sex differences in the clustering of metabolic syndrome factors: association with mortality risk. Diabetes Care. 2010;33(11):2457-2461.

4. Martin BC, et al. Role of glucose and insulin resistance in development of type 2 diabetes mellitus: results of a 25-year follow-up study. Lancet.
1992;340(8825):925-929.

5. Pucci G, et al. Sex- and gender-related prevalence, cardiovascular risk and therapeutic approach in metabolic syndrome: a review of the literature. Pharmacol Res. 2017;120:34-42.

6. Bogardus C, et al. Relationships between insulin secretion, insulin action, and fasting plasma glucose concentration in nondiabetic and noninsulin-dependent diabetic subjects. JClin Invest. 1984;74(4):1238-1246.

7. Hollenbeck C, Reaven GM. Variations in insulinstimulated glucose uptake in healthy individuals with normal glucose tolerance. J Clin Endocrinol
Metab. 1987;64(6):1169-1173.

8. Reaven GM. Pathophysiology of insulin resistance in human disease. Physiol Rev. 1995;75(3):473-486.

9. Goldfine $A B$, et al. Insulin resistance is a poor predictor of type 2 diabetes in individuals with no family history of disease. Proc Natl Acad Sci U S A. 2003;100(5):2724-2729.

10. Boucher J, et al. Insulin receptor signaling in normal and insulin-resistant states. Cold Spring Harb Perspect Biol. 2014;6(1):a009191.

11. Taniguchi CM, et al. Critical nodes in signalling pathways: insights into insulin action. Nat Rev 
Mol Cell Biol. 2006;7(2):85-96.

12. Dibble CC, Cantley LC. Regulation of mTORC1 by PI3K signaling. Trends Cell Biol. 2015;25(9):545-555.

13. Sakamoto K, Holman GD. Emerging role for AS160/TBC1D4 and TBC1D1 in the regulation of GLUT4 traffic. Am J Physiol Endocrinol Metab. 2008;295(1):E29-E37.

14. Ciaraldi TP, et al. GSK-3beta and control of glucose metabolism and insulin action in human skeletal muscle. Mol Cell Endocrinol. 2010;315(1-2):153-158.

15. Sajan MP, et al. AICAR and metformin, but not exercise, increase muscle glucose transport through AMPK-, ERK-, and PDK1-dependent activation of atypical PKC. Am J Physiol Endocrinol Metab. 2010;298(2):E179-E192.

16. O Sullivan IS, et al. FoxO1 integrates direct and indirect effects of insulin on hepatic glucose production and glucose utilization. Nat Commun. 2015;6:7079.

17. Sakaguchi M, et al. FoxK1 and FoxK2 in insulin regulation of cellular and mitochondrial metabolism. Nat Commun. 2019;10(1):1582.

18. Petersen MC, Shulman GI. Mechanisms of insulin action and insulin resistance. Physiol Rev. 2018;98(4):2133-2223.

19. Abdul-Ghani MA, DeFronzo RA. Pathogenesis of insulin resistance in skeletal muscle. J Biomed Biotechnol. 2010;2010:476279.

20. Batista TM, et al. A cell-autonomous signature of dysregulated protein phosphorylation underlies muscle insulin resistance in type 2 diabetes. Cell Metab. 2020;32(5):844-859.

21. Burkart AM, et al. Insulin resistance in human ips cells reduces mitochondrial size and function. $\mathrm{Sci}$ Rep. 2016;6:22788.

22. Iovino $S$, et al. Genetic insulin resistance is a potent regulator of gene expression and proliferation in human iPS cells. Diabetes. 2014;63(12):4130-4142.

23. Iovino $S$, et al. Myotubes derived from humaninduced pluripotent stem cells mirror in vivo insulin resistance. Proc Natl Acad Sci U S A. 2016;113(7):1889-1894.

24. Carcamo-Orive I, et al. Analysis of transcriptional variability in a large human iPSC library reveals genetic and non-genetic determinants of heterogeneity. Cell Stem Cell. 2017;20(4):518-532.

25. Caron L, et al. A human pluripotent stem cell model of facioscapulohumeral muscular dystrophy-affected skeletal muscles. Stem Cells Transl Med. 2016;5(9):1145-1161.

26. Massart J, et al. Altered miR-29 expression in type 2 diabetes influences glucose and lipid metabolism in skeletal muscle. Diabetes. 2017;66(7):1807-1818.

27. Langfelder P, et al. Defining clusters from a hierarchical cluster tree: the Dynamic Tree Cut package for R. Bioinformatics. 2008;24(5):719-720.

28. Li Y, et al. Protein kinase C Theta inhibits insulin signaling by phosphorylating IRS1 at Ser(1101). J Biol Chem. 2004;279(44):45304-45307.

29. Tremblay F, et al. Identification of IRS-1 Ser-1101 as a target of S6K1 in nutrient- and obesityinduced insulin resistance. Proc Natl Acad Sci U S A. 2007;104(35):14056-14061.

30. Bhatt $\mathrm{S}$, et al. Preserved DNA damage check- point pathway protects against complications in long-standing type 1 diabetes. Cell Metab. 2015;22(2):239-252.

31. Kubo K, et al. A novel, sensitive, and specific assay for abasic sites, the most commonly produced DNA lesion. Biochemistry. 1992;31(14):3703-3708.

32. Moller LLV, et al. Rho GTPases-emerging regulators of glucose homeostasis and metabolic health. Cells. 2019;8(5):E434.

33. Batista TM, et al. Defining the underlying defect in insulin action in type 2 diabetes. Diabetologia. 2021;64(5):994-1006.

34. Copps KD, White MF. Regulation of insulin sensitivity by serine/threonine phosphorylation of insulin receptor substrate proteins IRS1 and IRS2. Diabetologia. 2012;55(10):2565-2582.

35. Yoshitomi $\mathrm{H}$, et al. L-Citrulline increases hepatic sensitivity to insulin by reducing the phosphorylation of serine 1101 in insulin receptor substrate-1. BMC Complement Altern Med. 2015;15:188.

36. Bellacosa A, et al. Akt activation by growth factors is a multiple-step process: the role of the $\mathrm{PH}$ domain. Oncogene. 1998;17(3):313-325.

37. Liao Y, Hung MC. Physiological regulation of Akt activity and stability. Am J Transl Res. 2010;2(1):19-42.

38. Joshi J, et al. Par- 4 inhibits Akt and suppresses Ras-induced lung tumorigenesis. $E M B O \mathrm{~J}$. 2008;27(16):2181-2193.

39. Soliman GA, et al. mTOR Ser-2481 autophosphorylation monitors mTORC-specific catalytic activity and clarifies rapamycin mechanism of action. J Biol Chem. 2010;285(11):7866-7879.

40. Zhang X, et al. Amino acids-Rab1A-mTORC1 signaling controls whole-body glucose homeostasis. Cell Rep. 2021;34(11):108830.

41. Laplante M, Sabatini DM. mTOR signaling in growth control and disease. Cell. 2012;149(2):274-293.

42. Treebak JT, et al. Acute exercise and physiological insulin induce distinct phosphorylation signatures on TBC1D1 and TBC1D4 proteins in human skeletal muscle. J Physiol. 2014;592(2):351-375.

43. He L, et al. mTORC1 promotes metabolic reprogramming by the suppression of GSK3dependent Foxk1 phosphorylation. Mol Cell. 2018;70(5):949-960.

44. Batista TM, et al. Multi-dimensional transcriptional remodeling by physiological insulin in vivo. Cell Rep. 2019;26(12):3429-3443.

45. Pihlajamaki J, et al. Expression of the splicing factor gene SFRS10 is reduced in human obesity and contributes to enhanced lipogenesis. Cell Metab. 2011;14(2):208-218

46. Lee SC, Chan JC. Evidence for DNA damage as a biological link between diabetes and cancer. Chin Med J (Engl). 2015;128(11):1543-1548.

47. Oliva M, et al. The impact of sex on gene expression across human tissues. Science. 2020;369(6509):eaba3066.

48. CDC. Prevalence of Both Diagnosed and Undiagnosed Diabetes.https://www.cdc.gov/diabetes/ data/statistics-report/diagnosedundiagnosed-diabetes.html. Updated June 24, 2020. Accessed August 31, 2021.
49. CDC. Prevalence of Obesity and Severe Obesity Among Adults: United States, 2017-2018. https://www.cdc.gov/nchs/products/databriefs/ db360.htm. Updated February 27, 2020. Accessed August 31, 2021

50. Lundsgaard AM, Kiens B. Gender differences in skeletal muscle substrate metabolism - molecular mechanisms and insulin sensitivity. Front Endocrinol (Lausanne). 2014;5:195.

51. Perreault $\mathrm{L}$, et al. Sex differences in diabetes risk and the effect of intensive lifestyle modification in the Diabetes Prevention Program. Diabetes Care. 2008;31(7):1416-1421.

52. Dagogo-Jack $S$, et al. Lack of racial disparity in incident prediabetes and glycemic progression among black and white offspring of parents with type 2 diabetes: the pathobiology of prediabetes in a biracial cohort (POP-ABC) study. J Clin Endocrinol Metab. 2014;99(6):E1078-E1087.

53. Hilawe EH, et al. Differences by sex in the prevalence of diabetes mellitus, impaired fasting glycaemia and impaired glucose tolerance in sub-Saharan Africa: a systematic review and meta-analysis. Bull World Health Organ. 2013;91(9):671-682D.

54. Colhoun H. Coronary heart disease in women: why the disproportionate risk? Curr Diab Rep. 2006;6(1):22-28

55. Peters SA, et al. Diabetes as a risk factor for stroke in women compared with men: a systematic review and meta-analysis of 64 cohorts, including 775,385 individuals and 12,539 strokes. Lancet. 2014;383(9933):1973-1980.

56. Frobel J, et al. Epigenetic rejuvenation of mesenchymal stromal cells derived from induced pluripotent stem cells. Stem Cell Reports. 2014;3(3):414-422.

57. Knowles JW, et al. Measurement of insulinmediated glucose uptake: direct comparison of the modified insulin suppression test and the euglycemic, hyperinsulinemic clamp. Metabolism. 2013;62(4):548-553.

58. Humphrey SJ, et al. High-throughput and high-sensitivity phosphoproteomics with the EasyPhos platform. Nat Protoc. 2018;13(9):1897-1916.

59. Cox J, Mann M. MaxQuant enables high peptide identification rates, individualized p.p.b.-range mass accuracies and proteome-wide protein quantification. Nat Biotechnol. 2008;26(12):1367-1372.

60. Tyanova S, et al. The Perseus computational platform for comprehensive analysis of (prote)omics data. Nat Methods. 2016;13(9):731-740.

61. Ritchie ME, et al. limma powers differential expression analyses for RNA-sequencing and microarray studies. Nucleic Acids Res. 2015;43(7):e47.

62. Hornbeck PV, et al. PhosphoSitePlus, 2014: mutations, PTMs and recalibrations. Nucleic Acids Res. 2015;43(database issue):D512-D520.

63. Lee TY, et al. RegPhos: a system to explore the protein kinase-substrate phosphorylation network in humans. Nucleic Acids Res. 2011;39(database issue):D777-D787.

64. Leek JT, Storey JD. Capturing heterogeneity in gene expression studies by surrogate variable analysis. PLoS Genet. 2007;3(9):1724-1735. 